\title{
О ЧЕМ И ПОЧЕМУ МОЛЧАТ АБОРИГЕНЫ
}

\section{Natal'ja Novikova}

\section{OpenEdition}

\section{Journals}

Édition électronique

URL : https://journals.openedition.org/efo/5009

DOI : 10.4000/efo.5009

ISSN : 2275-1947

Éditeur

INALCO

Édition imprimée

ISBN : 978-2-343-08571-5

ISSN : 0071-2051

Référence électronique

Natal'ja Novikova, « О чем и почему молчат аборигены », Études finno-ougriennes [En ligne], 47 |

2015, mis en ligne le 16 juin 2016, consulté le 20 septembre 2021. URL : http://

journals.openedition.org/efo/5009; DOI : https://doi.org/10.4000/efo.5009

Ce document a été généré automatiquement le 20 septembre 2021

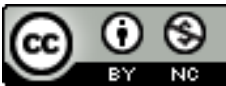

Études finno-ougriennes est mis à disposition selon les termes de la Licence Creative Commons Attribution - Pas d'Utilisation Commerciale 4.0 International. 


\title{
О ЧЕМ И ПОЧЕМУ МОЛЧАТ
}

\section{АБОРИГЕНЫ}

\author{
Natal'ja Novikova
}

\section{NOTE DE L'AUTEUR}

РАБОТА ВЫПОЛНЕНА В РАМКАХ ПРОГРАММЫ ФУНДАМЕНТАЛЬНЫХ ИССЛЕДОВАНИЙ ПРЕЗИДИУМА РАН «ПИСКОВЫЕ ФУНДАМЕНТАЛЬНЫЕ НАУЧНЫЕ ИССЛЕДОВАНИЯ В ИНТЕРЕСАХ РАЗВИТИЯ АРКТИЧЕСКОЙ ЗОНЫ РОССИЙСКОЙ ФЕДЕРАЦИИ» (ПРОЕКТ «КОРЕННЫЕ НАРОДЫ И ПРОМЫШЛЕННОЕ ОСВОЕНИЕ АРКТИКИ: ПРЕОДОЛЕНИЕ РИСКОВ И СТРАТЕГИИ РАЗВИТИЯ»).

1 СЛОВО И МОЛЧАНИЕ В МИРЕ КОРЕННЫХ НАРОДОВ ВЫПОЛНЯЮТ КОММУНИКАТИВНУЮ ФУНКЦИЮ, ПРИЧЕМ ВО МНОГИХ ВАЖНЫХ СИТУАЦИЯХ ПРИОРИТЕТ ПРИНАДЛЕЖИТ ИМЕННО МОЛЧАНИЮ. МОЛЧАНИЕ ВЫСТУПАЕТ И КУЛЬТУРНЫМ МАРКЕРОМ АБОРИГЕНОВ, ВАЖНЕЙШИМ КОДОМ И СРЕДСТВОМ ИХ ПРЕЗЕНТАЦИИ В СОВРЕМЕННОМ МИРЕ. МОЛЧАНИЕ ЯВЛЯЕТСЯ СВОЕОБРАЗНЫМ ПАРОЛЕМ ВО ВЗАИМОДЕЙСТВИИ КОРЕННЫХ НАРОДОВ С ОКРУЖАЮЩИМ МИРОМ, НО ЧТОБЫ ПОНЯТЬ ЕГО И СУМЕТЬ ИНТЕРПРЕТИРОВАТЬ, НЕОБХОДИМО ИССЛЕДОВАТЬ КУЛЬТУРУ АБОРИГЕНОВ, ПОНЯТЬ, ПОЧЕМУ И КОГДА ОНИ ГОВОРЯТ ИЛИ МОЛЧАТ. МОЛЧАНИЕ МОЖНО СРАВНИТЬ С ВЕЧНОЙ МЕРЗЛОТОЙ, КОТОРАЯ ЯВЛЯЕТСЯ ОСНОВОЙ СОХРАНЕНИЯ АРКТИЧЕСКОЙ ПРИРОДЫ. В ВОСПРИЯТИИ ЖЕ ПРЕДСТАВИТЕЛЕЙ ДРУГИХ КУЛЬТУР МОЛЧАНИЕ ИЛИ НЕДОГОВОРЕННОСТИ АБОРИГЕНОВ ЧАСТО ВОСПРИНИМАЮТСЯ КАК НЕДОСТАТОК послЕДНИХ.

\section{Право на слово и молчание}

2 РЕЧЬ И МОЛЧАНИЕ ВЫСТУПАЮТ НЕРАЗРЫВНЫМИ ХАРАКТЕРИСТИКАМИ МИРА КОРЕННЫХ НАРОДОВ СЕВЕРА. ОСОБЕННО ЯРКО ОНИ ПРОЯВЛЯЮТСЯ В СФЕРЕ ИХ 
ВЗАИМОДЕЙСТВИЯ С ПРОМЫШЛЕННЫМИ КОРПОРАЦИЯМИ И ОРГАНАМИ ГОСУДАРСТВЕННОЙ ВЛАСТИ. ИМЕННО С ЦЕЛЬЮ ДАТЬ АБОРИГЕНАМ ПРАВО ГОЛОСА В ГОСУДАРСТВЕННОЙ ПРАВОВОЙ СИСТЕМЕ И БЫЛИ ПРИНЯТО ПОСВЯЩЕННОЕ ИМ ЗАКОНОДАТЕЛЬСТВО В РОССИИ. БЕЗ ЭТОГО ИХ ЧАЯНИЯ И ИНТЕРЕСЫ ОТНОСИЛИСЬ К СФЕРЕ МОЛЧАНИЯ СО СТОРОНЫ ГОСУДАРСТВЕННЫХ ОРГАНОВ. И ЭТО МОЛЧАНИЕ ЗНАЧИЛО ПУСТОТУ. ОНО СОЗДАВАЛО ПРОПАСТЬ МЕЖДУ КОРЕННЫМИ НАРОДАМИ И ПРАВИТЕЛЬСТВОМ, ЧЕЛОВЕКОМ И ГОСУДАРСТВОМ. КОРЕННЫЕ МАЛОЧИСЛЕННЫЕ НАРОДЫ В РОССИИ ВЫШЛИ ИЗ ЭТОГО МОЛЧАНИЯ В 1993 ГОДУ, КОГДА БЫЛА ПРИНЯТА НОВАЯ КОНСТИТУЦИЯ, ПОТОМ БЫЛИ ПРИНЯТЫ ЗАКОНЫ, РЕГЛАМЕНТИРУЮЩИЕ ИХ ПОЛОЖЕНИЕ В ГОСУДАРСТВЕ, НО И СЕГОДНЯ МНОГИЕ ПРОБЛЕМЫ ДАЛЕКИ ОТ РАЗРЕШЕНИЯ. В ДАННОЙ СТАТЬЕ Я ХОЧУ ОСТАНОВИТЬСЯ ТОЛЬКО НА ОДНОМ АСПЕКТЕ АНТРОПОЛОГИИ МОЛЧАНИЯ, В КОНТЕКСТЕ ГОСУДАРСТВЕННОЙ ПОЛИТИКИ, СВЯЗАННОМ С СУДЕБНОЙ ПРАКТИКОЙ. СКАЗАННОЕ ИЛИ НАПИСАННОЕ СЛОВО ВОСПРИНИМАЕТСЯ КАК НОРМА ЖЕСТКОЙ РЕГЛАМЕНТАЦИИ И ЧАСТО НЕ СООТВЕТСТВУЕТ МЕХАНИЗМАМ ПЕРЕДАЧИ ИНФОРМАЦИИ, ПРИНЯТЫМ В ТРАДИЦИОННЫХ ОБЩЕСТВАХ. ЭТА ЖЕ ЧЕРТА КУЛЬТУРЫ ХАРАКТЕРИЗУЕТ ПОВЕДЕНИЕ АБОРИГЕНОВ В СУДЕ, НЕВОЗМОЖНОСТЬ ДЛЯ НИХ ГОВОРИТЬ О НЕКОТОРЫХ ВЕЩАХ. В СОВРЕМЕННЫХ УСЛОВИЯХ ЭТО МОЖЕТ БЫТЬ СВЯЗАНО С ИХ НЕОПЫТНОСТЬЮ И ПРАВОВОЙ НЕГРАМОТНОСТЬЮ. КАК И БОЛЬШИНСТВО ЛЮДЕЙ В РОССИИ, ОНИ ИСПЫТЫВАЮТ НЕДОСТАТОК ПРАВОВЫХ ЗНАНИЙ И НАВЫКОВ ИХ ПРИМЕНЕНИЯ. ВМЕСТЕ С ТЕМ, В КУЛЬТУРЕ АБОРИГЕНОВ ЕСТЬ МНОГО ЗАПРЕТОВ, КОГДА НЕЛЬЗЯ, НАПРИМЕР, ГОВОРИТЬ О МАЛЕНЬКИХ ДЕТЯХ ИЛИ О СВЯЩЕННЫХ МЕСТАХ. СОГЛАСНО СТАТЬЕ 14 ФЕДЕРАЛЬНОГО ЗАКОНА «О ГАРАНТИЯХ ПРАВ КОРЕННЫХ МАЛОЧИСЛЕННЫХ НАРОДОВ РОССИЙСКОЙ ФЕДЕРАЦИИ»,

ПРИ РАССМОТРЕНИИ В СУДАХ ДЕЛ, В КОТОРЫХ ЛИЦА, ОТНОСЯЩИЕСЯ К МАЛОЧИСЛЕННЫМ НАРОДАМ, ВЫСТУПАЮТ В КАЧЕСТВЕ ИСТЦОВ, ОТВЕТЧИКОВ, ПОТЕРПЕВШИХ ИЛИ ОБВИНЯЕМЫХ, МОГУТ ПРИНИМАТЬСЯ ВО ВНИМАНИЕ ТРАДИЦИИ И ОБЫЧАИ ЭТИХ НАРОДОВ, НЕ ПРОТИВОРЕЧАЩИЕ ФЕДЕРАЛЬНЫМ ЗАКОНАМ И ЗАКОНАМ СУБЪЕКТОВ РОССИЙСКОЙ ФЕДЕРАЦИИ. В ЦЕЛЯХ ЭФФЕКТИВНОЙ СУДЕБНОЙ ЗАЩИТЫ ПРАВ МАЛОЧИСЛЕННЫХ НАРОДОВ ДОПУСКАЕТСЯ УЧАСТИЕ В УКАЗАННОЙ СУДЕБНОЙ ЗАЩИТЕ УПОЛНОМОЧЕННЫХ ПРЕДСТАВИТЕЛЕЙ МАЛОЧИСЛЕННЫХ НАРОДОВ.

В ТАКИХ СЛУЧАЯХ АНТРОПОЛОГ МОЖЕТ ВЫСТУПИТЬ ЭКСПЕРТОМ-ПОСРЕДНИКОМ В СУДЕ, ДАТЬ НЕОБХОДИМЫЕ ПОЯСНЕНИЯ, ВЕДЬ ТОЛЬКО СПЕЦИАЛИСТ МОЖЕТ ОБЪЯСНИТЬ СУДУ СУТЬ ТАКИХ УМОЛЧАНИЙ. ПРИ ЭТОМ НУЖНО ЗНАТЬ, ЧТО СУДЕБНОЕ ЗАСЕДАНИЕ ПО ПРОСЬБЕ УЧАСТНИКОВ ПРОЦЕССА МОЖЕТ БЫТЬ В ОСОБЫХ СЛУЧАЯХ ЗАКРЫТЫМ И МОЖНО ВОСПОЛЬЗОВАТЬСЯ ЭТИМ ПРАВОМ.

ПРАВДА, ПОКА ТАКИХ СУДЕБНЫХ ПРОЦЕССОВ СРАВНИТЕЛЬНО НЕМНОГО. ИХ АНАЛИЗ ПРОЦЕССОВ ПОКАЗЫВАЕТ, ЧТО ОСОБЕННОСТИ ТРАДИЦИОННОГО ОБРАЗА ЖИЗНИ, АБОРИГЕННОГО МИРОВОЗЗРЕНИЯ, ЧАСТО СТАНОВЯТСЯ ПРЕПЯТСТВИЕМ ДЛЯ ВЫНЕСЕНИЯ СПРАВЕДЛИВОГО РЕШЕНИЯ, ТАК КАК ПРОСТО ОСТАЮТСЯ НЕПОНЯТЫМИ СУДЬЯМИ. РАЗЛИЧИЯ В СРЕДСТВАХ КОММУНИКАЦИИ УЧАСТНИКОВ ТАКИХ ПРОЦЕССОВ СОЗДАЮТ ДОПОЛНИТЕЛЬНЫЕ СЛОЖНОСТИ, НО ОНИ НЕОТЪЕМЛЕМЫ ОТ НОРМАТИВНОЙ КУЛЬТУРЫ И ДОЛЖНЫ СТАТЬ ПРЕДМЕТОМ ИЗУЧЕНИЯ НЕ ТОЛЬКО АНТРОПОЛОГОВ, НО И ЮРИСТОВ.

МЕЖДУНАРОДНОЕ ПРАВО БОЛЕЕ ЧУВСТВИТЕЛЬНО К ОСОБЕННОСТЯМ КОММУНИКАЦИИ В КУЛЬТУРЕ КОРЕННЫХ НАРОДОВ. МОЛЧАНИЕ РАССМАТРИВАЕТСЯ КАК ЧАСТЬ МИРА КОРЕННЫХ НАРОДОВ. В 1992 ГОДУ БЫЛА ПРИНЯТА КОНВЕНЦИЯ ООН О БИОРАЗНООБРАЗИИ, КОТОРАЯ ПРЕДУСМАТРИВАЕТ ОБЯЗАННОСТИ ГОСУДАРСТВ- 
УЧАСТНИКОВ В ОБЛАСТИ СОХРАНЕНИЯ И ПОДДЕРЖАНИЯ ТРАДИЦИОННЫХ ЗНАНИЙ И ПРАКТИК КОРЕННЫХ НАРОДОВ, ИМЕЮЩИХ ЗНАЧЕНИЕ ДЛЯ СОХРАНЕНИЯ БИОЛОГИЧЕСКОГО РАЗНООБРАЗИЯ. РЕЗУЛЬТАТОМ РАБОТЫ КОНФЕРЕНЦИИ ПО ЭТОЙ КОНВЕНЦИИ СТАЛИ «ДОБРОВОЛЬНЫЕ РУКОВОДЯЩИЕ ПРИНЦИПЫ АГУЭЙ-ГУ ПРОВЕДЕНИЯ ОЦЕНОК КУЛЬТУРНЫХ, ЭКОЛОГИЧЕСКИХ И СОЦИАЛЬНЫХ ПОСЛЕДСТВИЙ ПРЕДЛАГАЕМОЙ РЕАЛИЗАЦИИ ПРОЕКТОВ В МЕСТАХ РАСПОЛОЖЕНИИ СВЯТЫНЬ, А ТАКЖЕ НА ЗЕМЛЯХ И В АКВАТОРИЯХ, ЗАНИМАЕМЫМ ИЛИ ИСПОЛЬЗУЕМЫМ КОРЕННЫМИ И МЕСТНЫМИ ОБЩИНАМИ». В ЭТОМ ДОКУМЕНТЕ ОЦЕНКА КУЛЬТУРНЫХ ПОСЛЕДСТВИЙ В ТОМ ЧИСЛЕ СТРОИТСЯ В СООТВЕТСТВИИ С УВАЖЕНИЕМ ПОТРЕБНОСТИ В УЕДИНЕНИИ ДЛЯ ЦЕЛЕЙ, СВЯЗАННЫХ С КУЛЬТУРОЙ, ЧТО МОЖНО ИНТЕРПРЕТИРОВАТЬ КАК ГАРАНТИЯ ПРАВА НА МОЛЧАНИЕ.

6 УСТОЙЧИВОСТЬ КОММУНИКАТИВНЫХ ГРАНИЦ ЯВЛЯЕТСЯ ОДНОЙ ИЗ ПРИЧИН ТОГО, ЧТО КОРЕННЫЕ НАРОДЫ ЧАСТО НЕ ВОСПРИНИМАЮТ «НАПИСАННЫЙ» ЗАКОН, А ПРОДОЛЖАЮТ СТРОИТЬ СВОЮ ЖИЗНЬ ПО НОРМАМ ОБЫЧНОГО ПРАВА, КОТОРОЕ НЕ ПОДДАЕТСЯ ПИСЬМЕННОЙ ФИКСАЦИИ. ОСОБЫЙ ИНТЕРЕС В СОВРЕМЕННОМ МИРЕ ПРЕДСТАВЛЯЮТ АЛЬТЕРНАТИВНЫЕ ВНЕСУДЕБНЫЕ ПРОЦЕДУРЫ РАЗРЕШЕНИЯ КОНФЛИКТОВ, КОТОРЫЕ ПРИНЯТЫ НЕ ТОЛЬКО В АБОРИГЕННОМ СООБЩЕСТВЕ, НО И НЕКОТОРЫМИ ГОСУДАРСТВАМИ, НАПРИМЕР, В ЮВЕНАЛЬНОЙ ЮСТИЦИИ КАНАДЫ (ПОДРОБНЕЕ: НОВИКОВА 2007, С. 111-128). ВАЖНЕЙШЕЙ ЧЕРТОЙ АБОРИГЕННОГО РАЗРЕШЕНИЯ КОНФЛИКТОВ ЯВЛЯЕТСЯ НЕОБХОДИМОСТЬ ДОСТИЖЕНИЯ КОНСЕНСУСА. А ОДНИМ ИЗ СРЕДСТВ ЭТОГО - ОРЛИНОЕ ПЕРО КАК СИМВОЛ СЛОВА В КРУГАХ ПРАВОСУДИЯ, КОТОРЫЕ ПРОВОДЯТСЯ В СЕВЕРНОЙ АМЕРИКЕ И КАНАДЕ. В НИХ ЛЮДИ ВЫСКАЗЫВАЮТСЯ, ТОЛЬКО ВЗЯВ В РУКИ ТАКОЕ ПЕРО, ЕГО ПЕРЕДАЮТ ПО ЧАСОВОЙ СТРЕЛКЕ. СИМВОЛ СЛОВА ПОМОГАЕТ ГОВОРИТЬ И СЛУШАТЬ, ОН СОЗДАЕТ ОПРЕДЕЛЕННЫЙ РИТМ ДИАЛОГА И ОБЕСПЕЧИВАЕТ РАВЕНСТВО В КРУГЕ. СИМВОЛОМ СЛОВА МОЖЕТ БЫТЬ И ДРУГОЙ ПРЕДМЕТ, НО ОН ДОЛЖЕН ОБЯЗАТЕЛЬНО ПОЛЬЗОВАТЬСЯ УВАЖЕНИЕМ УЧАСТНИКОВ КРУГА, ИМЕТЬ СИМВОЛИЧЕСКУЮ НАГРУЗКУ (ПРАНИС, СТЮАРТ, УЕДЖ 2010, С.94-103). ТАКИМ ОБРАЗОМ, ЗНАК СЛОВА СТАНОВИТСЯ ОПРЕДЕЛЯЮЩИМ В ОБЩЕНИИ, ВЕДЬ ОН РЕГУЛИРУЕТ НЕ ТОЛЬКО ПРАВО ГОВОРИТЬ, КОГДА ТЫ ДЕРЖИШЬ ЭТО ПЕРО В РУКАХ, НО И ОБЯЗАННОСТЬ ВСЕХ ОСТАЛЬНЫХ МОЛЧАТЬ. БЛАГОДАРЯ СИМВОЛУ СЛОВА ЛЮДИ НЕ ПЕРЕБИВАЮТ ДРУГ ДРУГА, У КАЖДОГО ПОЯВЛЯЕТСЯ ВОЗМОЖНОСТЬ БЫТЬ УСЛЫШАННЫМ, А ЗНАЧИТ В РЕЗУЛЬТАТЕ КОНФЛИКТ БУДЕТ УРЕГУЛИРОВАН.

7 СХОДНЫЕ ПРЕДСТАВЛЕНИЯ СУЩЕСТВУЮТ И КОРЕННЫХ НАРОДОВ СЕВЕРА. ВО МНОГИХ СИТУАЦИЯХ НЕМНОГОСЛОВНОСТЬ АБОРИГЕНОВ СЕВЕРА ТАКЖЕ СОЗДАЕТ ВОЗМОЖНОСТЬ ЛУЧШЕ СЛЫШАТЬ ДРУГ ДРУГА. ИНТЕРЕСНОЕ НАБЛЮДЕНИЕ СОДЕРЖИТСЯ В РАБОТЕ ПО САМОДИЙСКИМ КУЛЬТУРАМ О.Б. ХРИСТОФОРОВОЙ:

ДАЖЕ ЧТО-ЛИБО ВАЖНОЕ ЧЕЛОВЕК НЕ МОЖЕТ СКАЗАТЬ, НЕ ЗАРУЧИВШИСЬ ПРЕЖДЕ СОГЛАСИЕМ СЛУШАТЕЛЯ: КЛИШЕ “У МЕНЯ ЕСТЬ СЛОВО” ОЗНАЧАЕТ ПРОСЬБУ ВЫСЛУШАТЬ, И ПОКА СОБЕСЕДНИК НЕ ПРОИЗНЕСЕТ “ОТЗЫВ”: “КАКОЕ СЛОВО, ГОВОРИ”, НИЧЕГО НЕ МОЖЕТ БЫТЬ СКАЗАНО. В ЦЕЛОМ, САМОДИЙСКИЕ КУЛЬТУРЫ МОЖНО НАЗВАТЬ “МОЛЧАЩИМИ". НОРМЫ ИХ РЕЧЕВОГО ЭТИКЕТА НЕ ДОПУСКАЮТ ПРЯМОГО ВЫРАЖЕНИЯ ПРОСЬБЫ, ОТКАЗА И ОЦЕНКИ. ПРЕДПОЧТЕНИЕ НЕКАТЕГОРИЧНЫХ ФОРМ ВЫСКАЗЫВАНИЯ, НЕВЕРБАЛЬНЫХ СРЕДСТВ И ИНОСКАЗАНИЙ ОБЪЯСНЯЕТСЯ СТРЕМЛЕНИЕМ НЕ НАВЯЗАТЬ СВОЕГО МНЕНИЯ СОБЕСЕДНИКУ, ДАТЬ ЕМУ ВОЗМОЖНОСТЬ ПОНЯТЬ ПОДТЕКСТ ВЫСКАЗЫВАНИЯ И САМОМУ СДЕЛАТЬ ВЫВОДЫ. ГОВОРЯ ЧТО-ЛИБО (ВНЕ ЗАВИСИМОСТИ ОТ КОДА, 
КОТОРЫМ ВЫРАЖЕНО СООБЩЕНИЕ), ЕГО ОТПРАВИТЕЛЬ НАДЕЕТСЯ, ЧТО ПОЛУЧАТЕЛЬ ОТВЕТИТ НУЖНЫМ ОБРАЗОМ. НА СВОИ ДЕЙСТВИЯ ЧЕЛОВЕК СМОТРИТ ГЛАЗАМИ ПАРТНЕРА ПО ВЗАИМОДЕЙСТВИЮ; ТАКОГО ЖЕ РЕФЛЕКСИВНОГО - И СОЦИАЛЬНО ОТВЕТСТВЕННОГО - ПОВЕДЕНИЯ ОЖИДАЕТ И ОТ НЕГО (ХРИСТОФОРОВА 2006, c. 82-194). КУЛЬТУРНАЯ ГРАНИЦА. ХОТЯ МОИ ИНФОРМАНТЫ ГОВОРИЛИ О ВОЗМОЖНОСТИ «ОБЩАТЬСЯ МОЛЧА» И С ПРЕДСТАВИТЕЛЯМИ ДРУГИХ КУЛЬТУР, ОНИ ОТМЕЧАЛИ БОЛЬШУЮ РОЛЬ МОЛЧАНИЯ В СВОЕЙ КУЛЬТУРЕ. В ИНТЕРВЬЮ С ХУДОЖНИКОМ Г.С. РАЙШЕВЫМ Я ЗАПИСАЛА ЕГО МНЕНИЕ О РАЗНЫХ ТИПАХ МОЛЧАНИЯ:

КУЛЬТУРА МОЛЧАНИЯ - ЕСТЬ У НАРОДА, НЕ ГОВОРИТЬ ЛИШНЕЕ И НЕНУЖНОЕ, А СЛУШАТЬ И СООТНОСИТСЯ ЧАСТО НЕ СЛОВАМИ, А СВОИМ ПОВЕДЕНИЕМ, ОТНОШЕНИЕМ. ВОТ С ОДНИМ ЧЕЛОВЕКОМ ВСТРЕЧАЕШЬСЯ, ТЫ НАПРЯЖЕН, А С ДРУГИМ - НЕТ НАПРЯЖЕНИЯ, ЗНАЧИТ, ЕСТЬ КУЛЬТУРА ДОВЕРИТЕЛЬНОСТИ, ОНА СТАНОВИТСЯ НЕ СЛОВОМ, А МОЛЧАНИЕМ, МОЛЧАТЬ С ТАКИМ ЧЕЛОВЕКОМ МОЖНО. А КОГДА ТЫ ВСТРЕЧАЕШЬСЯ С ДРУГИМ ЧЕЛОВЕКОМ, МОЖЕТ ВОЗНИКНУТЬ ПСИХИЧЕСКОЕ НЕ РАВНОВЕСИЕ. ЕСЛИ ТЫ МОЛЧИШЬ, ТО ТЫ ДОЛЖЕН ДУМАТЬ. БЫВАЕТ АГРЕССИВНОЕ МОЛЧАНИЕ. ТАКИМ НАДЕЛЯЮТСЯ ЧИНОВНИКИ, И ЭТО НЕ СЛУЧАЙНО. ОНИ БУДТО БЫ БЕТОНИРУЮТ ПРОСТРАНСТВО ЭТИМ МОЛЧАНИЕМ. ЧЕЛОВЕК ПЕРЕД НИМИ СТАНОВИТСЯ РОБКИМ. ЭТО ОПРЕДЕЛЕННЫЙ СПОСОБ ВОЗДЕЙСТВИЯ НА ПРОСИТЕЛЯ. И Я БЫ ТАКИХ ЛЮДЕЙ СРАЗУ УВОЛЬНЯЛ» ${ }^{1}$.

МОЛЧАНИЕ ВЛАСТИ СЛУЖИТ ДРУГИМ ЦЕЛЯМ, УСТАНАВЛИВАЕМЫЕ В ЭТОМ СЛУЧАЕ ГРАНИЦЫ СТАНОВЯТСЯ НЕПРЕОДОЛИМЫМИ. НЕФТЯНИКИ ТАКЖЕ ИСПОЛЬЗУЮТ МОЛЧАНИЕ ДЛЯ ЗАКРЫТОСТИ, СОЗДАНИЯ ЗАМКНУТОЙ СУБКУЛЬТУРЫ. НАПРИМЕР, НЕФТЯНИКИ ОБЪЯВЛЯЮТ КАКУЮ-ТО ИНФОРМАЦИЮ КОММЕРЧЕСКОЙ ТАЙНОЙ, ЧТОБЫ СКРЫТЬ СВОИ ПЛАН ПО ИЗЪЯТИЮ ТЕРРИТОРИЙ ИЗ ТРАДИЦИОННОГО ПРИРОДОПОЛЬЗОВАНИЯ. В ЭТИХ СЛУЧАЯХ МОЛЧАНИЕ ОЗНАЧАЕТ ЗАКРЫТИЕ, ОТСУТСТВИЕ КОММУНИКАЦИИ, А РАЗРЕШЕНИЕ КОНФЛИКТА ВОЗМОЖНО ТОЛЬКО ГОСУДАРСТВЕННЫМИ СПОСОБАМИ - ЧЕРЕЗ СУД.

\section{Ты нечестно молчишь}

В ЭТНОГРАФИЧЕСКОЙ ЛИТЕРАТУРЕ МОЛЧАНИЕ РАССМАТРИВАЕТСЯ ЧАЩЕ ВСЕГО КАК ЗАПРЕТ СЛОВА, КАК ОБРАЗ ДРУГОГО. НОВЫЕ ПОЛЕВЫЕ МАТЕРИАЛЫ ПОЗВОЛЯЮТ ПО ИНОМУ ВЗГЛЯНУТЬ НА ЭТУ ПРОБЛЕМУ, ПОКАЗАТЬ РОЛЬ МОЛЧАНИЯ КАК СРЕДСТВА КОММУНИКАЦИИ. ИМЕННО ТАКАЯ ФУНКЦИЯ МОЛЧАНИЯ ЯВЛЯЕТСЯ ОПРЕДЕЛЯЮЩЕЙ В АБОРИГЕННОМ ОБЫЧНОМ ПРАВЕ. ПОКАЗАТЕЛЬНА В ЭТОМ ПЛАНЕ ФРАЗА В РАЗГОВОРЕ МЕЖДУ АБОРИГЕНАМИ - «ТЫ НЕ ЧЕСТНО МОЛЧИШЬ».

О НЕКОТОРЫХ ВЕЩАХ НЕЛЬЗЯ СКАЗАТЬ, НО МОЖНО ПЕРЕДАТЬ ИНФОРМАЦИЮ, ИСПОЛЬЗУЯ КАКИЕ-ТО ПРЕДМЕТЫ. БОЛЬШЕЕ ДОВЕРИЕ У АБОРИГЕНОВ ВЫЗЫВАЮТ НЕВЕРБАЛЬНЫЕ СРЕДСТВА ПЕРЕДАЧИ ИНФОРМАЦИИ, НАПРИМЕР, БИРКИ С НАСЕЧКАМИ. ТАК, НА ОДНОМ ИЗ ГЛАВНЫХ ПРАЗДНИКОВ ОБСКИХ УГРОВ - МЕДВЕЖЬЕМ, КОЛИЧЕСТВО ПРОПЕТЫХ ПЕСЕН ИЛИ ИСПОЛНЕННЫХ СЦЕН ФИКСИРУЕТСЯ НАСЕЧКАМИ НА СПЕЦИАЛЬНОЙ ПАЛОЧКЕ ИЛИ ПОСОХЕ. ПРИЧЕМ РАЗНЫЕ ПО ЗНАЧЕНИЮ И СТАТУСУ ПРЕДСТАВЛЕНИЯ ОПРЕДЕЛЯЛИСЬ РАЗНЫМ КОЛИЧЕСТВОМ НАСЕЧЕК. А ПОСЛЕДНИЕ, В СВОЮ ОЧЕРЕДЬ, УСИЛИВАЛИ ЗНАЧЕНИЕ ИСПОЛНЕННОГО НА ПРАЗДНИКЕ. 

ОНИ ОПОВЕЩАЮТ ОБ ЭТОМ СОРОДИЧЕЙ И СОСЕДЕЙ ТОЖЕ С ПОМОЩЬЮ ПАЛОЧКИ С НАСЕЧКАМИ. ПЕРЕДАДУТ ИЛИ ПОКАЖУТ ТЕБЕ ТАКУЮ ПАЛОЧКУ, УЗНАЕШЬ, КОГДА И КУДА ТЕБЕ НУЖНО ПРИЕХАТЬ. В 1931Г. С ПОМОЩЬЮ ПОДОБНОГО ПРЕДМЕТА СОБИРАЛИСЬ УЧАСТНИКИ КАЗЫМСКОГО ВОССТАНИЯ. ЭТО БЫЛА ШУМ-ТЫ-ЮХ - ПАЛКА, ПЕРЕДАВАВШАЯСЯ ИЗ СЕЛЕНИЯ В СЕЛЕНИЕ И ВОЗВРАЩАВШАЯСЯ К ОТПРАВИТЕЛЮ, НА КОТОРОЙ СТАВИЛИ ТАМГИ ВСЕ ЖЕЛАЮЩИЕ УЧАСТВОВАТЬ В СБОРЕ (ГОЛОВНЕВ 1995 , С. 167-168). ИНТЕРЕСНО, ЧТО ПОДОБНЫЕ БИРКИ ИСПОЛЬЗОВАЛИСЬ В ТУЗЕМНЫХ СУДАХ НА СЕВЕРЕ В ДВАДЦАТЫЕ-ТРИДЦАТЫЕ ГОДЫ, КОГДА СОВЕТСКАЯ ВЛАСТЬ ДЕЛАЛА ПОПЫТКУ ИСПОЛЬЗОВАТЬ НОРМЫ ОБЫЧНОГО ПРАВА ЭТИХ НАРОДОВ ПРИ СОЗДАНИИ СВОЕЙ СУДЕБНОЙ СИСТЕМЫ².

В НАУЧНОЙ ЛИТЕРАТУРЕ НЕВЕРБАЛЬНЫЕ СРЕДСТВА КОММУНИКАЦИИ ЧАЩЕ ВСЕГО ОПИСЫВАЮТСЯ ЧЕРЕЗ ЯЗЫК ЖЕСТОВ (НАПРИМЕР БУТОВСКАЯ 2004, КРЕЙДЛИН 2005). У АБОРИГЕНОВ СЕВЕРА ЖЕСТОВ СРАВНИТЕЛЬНО НЕМНОГО, ИХ КУЛЬТУРА ЛАКОНИЧНА В ЭТОМ ПЛАНЕ. В НЕЙ ПРИМЕНЯЮТСЯ СКОРЕЕ ДЕЙСТВИЯ, ЧЕМ ОТДЕЛЬНЫЕ ЖЕСТЫ. ТАК, НАПРИМЕР, ПРИГЛАШЕНИЕ ЗАЙТИ И ПОСИДЕТЬ В ЧУМЕ ДОСТАТОЧНО ШИРОКО ИСПОЛЬЗУЕТСЯ ДЛЯ УСТАНОВЛЕНИЯ КОНТАКТОВ. ОНИ ПРИВОЗЯТ ЧУМЫ В МОСКВУ И СТАВЯТ ИХ НА ПЛОЩАДИ НА ВВЦ, ТАКЖЕ ДЕЛАЮТ ВО ВРЕМЯ ПРОВЕДЕНИЯ ДНЯ ОЛЕНЕВОДА В ПОСЕЛКАХ И ГОРОДАХ НА СЕВЕРЕ. АБОРИГЕНЫ СЧИТАЮТ, ЧТО ЛЮДЯМ ДРУГИХ КУЛЬТУР ДОСТАТОЧНО ПРОСТО ПОСИДЕТЬ В ЧУМЕ, ВЫПИТЬ ЧАЮ ИЛИ ЧТО-ТО СЪЕСТЬ, И ОНИ СМОГУТ ПОЧУВСТВОВАТЬ КУЛЬТУРУ НАРОДОВ СЕВЕРА, А ЗНАЧИТ, ВПОСЛЕДСТВИИ, С НИМИ БУДЕТ ЛЕГЧЕ УСТАНОВИТЬ КОНТАКТ. ВОЗМОЖНО ПОЭТОМУ ОНИ В НАЧАЛЕ 2000-ХГГ. ШИРОКО ИСПОЛЬЗОВАЛИ ЧУМЫ В ПРОВЕДЕНИИ ПРЕДВЫБОРНОЙ КОМПАНИИ В ХАНТЫ-МАНСИЙСКОМ АВТОНОМНОМ ОКРУГЕ.

АОД̆СТВИЕ АБОРИГЕНОВ С РАБОТНИКАМИ ПРОМЫШЛЕННЫХ КОМПАНИЙ ПРОИСХОДИТ В БОЛЬШЕЙ СТЕПЕНИ В СФЕРЕ ДЕЛОВОГО ОБЩЕНИЯ - ПРИ ПОДПИСАНИИ ЭКОНОМИЧЕСКИХ СОГЛАШЕНИЙ, ВСТРЕЧАХ ПРИ ВОЗНИКНОВЕНИИ КОНФЛИКТОВ, ПОЭТОМУ ИНТЕРЕСНО ПРИВЕСТИ СЛЕДУЮЩИЕ ДАННЫЕ: ПСИХОЛОГИ РАЗНЫХ СТРАН ОТМЕЧАЮТ, ЧТО ВОЗДЕЙСТВИЕ, КОТОРОЕ МЫ В УСТНОМ ДЕЛОВОМ ОБЩЕНИИ ОКАЗЫВАЕМ НА ДРУГИХ ЛЮДЕЙ, ЗАВИСИТ ОТ ТОГО, ЧТО МЫ ГОВОРИМ, ЛИШЬ ПРИБЛИЗИТЕЛЬНО НА 7\%; ОТ ТОГО, КАК МЫ ГОВОРИМ, - НА 38\%, А ОТ ЯЗЫКА НАШЕГО ТЕЛА - НА 55\%. БЫЛО ТАКЖЕ ПОДСЧИТАНО, ЧТО 80\%, А ТО И 90\% ВСЕЙ ЭМОЦИОНАЛЬНОЙ ИНФОРМАЦИИ МЫ ЕЩЕ ДО СЛОВ ФАКТИЧЕСКИ ВЫРАЖАЕМ И ПЕРЕДАЕМ В ИНТЕРАКТИВНОЙ ДЕЛОВОЙ КОММУНИКАЦИИ НЕВЕРБАЛЬНЫМ СПОСОБОМ (КРЕЙДЛИН 2005, с. 152).

ПРИ ИЗУЧЕНИИ КОРЕННЫХ НАРОДОВ, ОСОБЕННО ИХ ВЗАИМОДЕЙСТВИЯ С ПРОМЫШЛЕННЫМИ КОМПАНИЯМИ И ОРГАНАМИ ГОСУДАРСТВЕННОЙ ВЛАСТИ, ОСОБЕННО ВАЖНО РАСКРЫТЬ СМЫСЛЫ ОГРАНИЧЕНИЙ НА СЛОВО, А ТАКЖЕ ПОКАЗАТЬ ИХ КУЛЬТУРНЫЙ КОНТЕКСТ. О.Б. ХРИСТОФОРОВА ОБРАТИЛА ВНИМАНИЕ НА ОСОБЕННОСТИ КОММУНИКАТИВНОГО ПОВЕДЕНИЯ НЕНЦЕВ КАК ВОЗМОЖНУЮ ПРИЧИНУ КОНФЛИКТОВ И ПРОБЛЕМ В ДЕЛОВЫХ КОНТАКТАХ МЕЖДУ АБОРИГЕНАМИ И ПРИЕЗЖАЮЩИМИ НА СЕВЕР РАЗЛИЧНЫМИ СПЕЦИАЛИСТАМИ, ПРИНЯВШИМИ УНИВЕРСАЛЬНУЮ СИСТЕМУ КОММУНИКАЦИИ, КОТОРАЯ У СЕВЕРЯН АССОЦИИРУЕТСЯ С РУССКИМИ. ТА ЖЕ НАПРЯЖЕННОСТЬ ХАРАКТЕРНА ДЛЯ ВЗАИМООТНОШЕНИЙ КОРЕННОГО НАСЕЛЕНИЯ С МЕСТНОЙ АДМИНИСТРАЦИЕЙ, ГОСУДАРСТВЕННЫМИ И КОММЕРЧЕСКИМИ ОРГАНИЗАЦИЯМИ. ПО МНЕНИЮ ХРИСТОФОРОВОЙ, ДАННОЕ ЯВЛЕНИЕ

Études finno-ougriennes, 47 | 2015 
ВО МНОГОМ ОБУСЛОВЛЕНО «ЭТНОКУЛЬТУРНОЙ СПЕЦИФИКОЙ НОРМАТИВНОГО КОММУНИКАТИВНОГО ПОВЕДЕНИЯ РУССКОГО И КОРЕННЫХ НАРОДОВ, ИНЫМИ СЛОВАМИ, НЕСОВПАДЕНИЕМ ПРЕДСТАВЛЕНИЙ О ПРАВИЛЬНОМ ПОВЕДЕНИИ ПРИ ОБЩЕНИИ». ОБ ОСОБОМ ОТНОШЕНИИ К СЛОВУ ГОВОРЯТ И ПОСЛОВИЦЫ КОРЕННЫХ НАРОДОВ, НАПРИМЕР:

«СЛОВО - ТОТ ЖЕ НОЖ БЕЗ НОЖЕН: БУДЬ С НИМ ОЧЕНЬ ОСТОРОЖЕН» (НГАНАСАНЫ); «КАК ОЛЕНЬ ВОЛКА БОИТСЯ, ТАК И ЛЮДИ БОЛТЛИВОГО ЧЕЛОВЕКА БОЯТСЯ» (НЕНЦЫ), СР. ЯКУТСКИЕ ПОСЛОВИЦЫ: «МЕНЬШЕ СЛОВ - СЛАДКО, МНОГО СЛОВ - ГОРЬКО»; «ЧЕЛОВЕЧЕСКОЕ СЛОВО - ЧТО СЕКИРА»; «ВО МНОГОСЛОВИИ МНОГО И ОШИБОК»; «СКАЗАННОЕ СЛОВО НЕ БЫВАЕТ НЕ УСЛЫШАННЫМ».(ЦИТИРУЕТСЯ ПО: ХРИСТОФОРОВА 1998, С. 224-231)

\section{О чем молчат}

НАРЯДУ С КУЛЬТУРОЙ РЕЧИ У АБОРИГЕНОВ СУЩЕСТВУЕТ КУЛЬТУРА МОЛЧАНИЯ. И ТО, О ЧЕМ И КАК МОЛЧАТ НА СТОЙБИЩАХ, МОЖЕТ МНОГОЕ СКАЗАТЬ О ТОМ, КАК АБОРИГЕНЫ УСТАНАВЛИВАЮТ МЕЖКУЛЬТУРНЫЕ КОММУНИКАЦИИ. ОСОБЕННО ВАЖНЫМ ЯВЛЯЕТСЯ ТО, ЧТО ЭТО ИМЕННО МЕЖКУЛЬТУРНЫЕ КОММУНИКАЦИИ, А НЕ ТОЛЬКО КОНТАКТ ВНУТРИ СООБЩЕСТВА. В СОВРЕМЕННЫХ УСЛОВИЯХ ЗНАЧЕНИЕ МОЛЧАНИЯ В ТАКОМ КАЧЕСТВЕ СТАНОВИТСЯ ВСЕ БОЛЕЕ ВАЖНЫМ И АКТУАЛЬНЫМ, ТАК КАК АБОРИГЕНЫ ВСЕ БОЛЬШЕ ВКЛЮЧАЮТСЯ В ЖИЗНЬ ОКРУЖАЮЩЕГО ИХ СООБЩЕСТВА. МОЖНО СКАЗАТЬ, ЧТО МОЛЧАНИЕ - ЭТО ЧАСТЬ ИХ ЗНАНИЙ ОБ ОКРУЖАЮЩЕМ МИРЕ, КОТОРЫЕ МОГУТ РАССМАТРИВАТЬСЯ КАК ВАЖНЕЙШИЙ КОМПОНЕНТ ИХ ИДЕНТИЧНОСТИ И СТАТУСА В ГОСУДАРСТВЕ.

ЗАПРЕТЫ НА СЛОВА, РЕЧЬ, НАЗЫВАНИЕ ЖИВОТНЫХ СТРОЯТСЯ У НЕКОТОРЫХ НАРОДОВ СЕВЕРА НА ПРЕДСТАВЛЕНИИ О СЛОВАХ И МЫСЛЯХ КАК РЕАЛЬНО СУЩЕСТВУЮЩИХ. ТАК, У ДОЛГАН, ПО ДАННЫМ А.А. ПОПОВА,

КАЖДЫЙ ОХОТНИК, ПОДСТЕРЕГАЮЩИЙ С РУЖЬЕМ НА ПОКОЛКЕ ДИКИХ ОЛЕНЕЙ, ЕСТЕСТВЕННО ДУМАЛ, КАК БЫ ДОБЫТЬ ОЛЕНЕЙ, Т.Е. ПО ЕГО ПРЕДСТАВЛЕНИЮ, ВСЕЛЯЛИСЬ В ОХОТНИКА ... ТЕНИ ОЛЕНЕЙ, СВЯЗАННЫЕ С ИХ МАТЕРИАЛЬНЫМ СУЩЕСТВОМ. И ЭТИ ОБРАЗЫ-ТЕНИ, ВЫХОДЯ ОБРАТНО ЧЕРЕЗ РОТ ОХОТНИКА, МОГЛИ ПРЕДУПРЕДИТЬ ОЛЕНЕЙ И НАПРАВИТЬ ИХ В ДРУГУЮ СТОРОНУ. ВО ИЗБЕЖАНИЕ ЭТОГО ОХОТНИК ЗАТЫКАЛ СЕБЕ РОТ ПУЧКОМ ТРАВЫ... А У ТАК НАЗЫВАЕМЫХ МЕДНЫХ ЭСКИМОСОВ СУЩЕСТВОВАЛ ЗАПРЕТ НА ТАНЦЫ И ПЕСНИ ВНЕ ДОМА, НА ОТКРЫТОМ ВОЗДУХЕ, ТАК КАК ПРИ ЭТОМ СЛОВА ПЕСНИ МОГЛИ «РАЗНЕСТИСЬ» В РАЗНЫЕ СТОРОНЫ, А ПЕВЦЫ УМЕРЕТЬ (ПОПОВ 1959, с. 89; Jenness 1923, p. 184; ЦИТ. по:, С. 119-126).

АНАЛИЗИРУЯ ЭТИ И МНОГОЧИСЛЕННЫЕ ДАННЫЕ ПО ДРУГИМ НАРОДАМ, С.В. ИВАНОВ ДЕЛАЕТ ВЫВОД О ТОМ, ЧТО СЛОВАМ В ТАКИХ СЛУЧАЯХ ПРИПИСЫВАЛАСЬ СПОСОБНОСТЬ ПРЕВРАЩАТЬСЯ В ВИДИМЫЕ ЖИВОТНЫЕ И ФАНТАСТИЧЕСКИЕ СУЩЕСТВА (ИВАНОВ 1975). КОНЕЧНО, ОПРЕДЕЛЕННЫЕ ТАБУ НА СЛОВА ИЛИ ОГРАНИЧЕНИЯ НА РЕЧЬ, ГОВОРЕНИЕ В ОПРЕДЕЛЕННЫХ СИТУАЦИЯХ ЕСТЬ У ВСЕХ НАРОДОВ. БОЛЬШАЯ РОЛЬ СЛОВА В КУЛЬТУРЕ АБОРИГЕНОВ ОПРЕДЕЛЯЕТСЯ ПРЕДСТАВЛЕНИЕМ О НЕМ КАК О НОСИТЕЛЕ ЖИЗНЕННОЙ СИЛЫ, ЭНЕРГИИ, НАПРИМЕР, У НЕНЦЕВ. СПЕЦИАЛИСТ ПО ФОЛЬКЛОРУ НЕНЦЕВ Е.Т. ПУШКАРЕВА ОТМЕЧАЕТ, ЧТО ОБРАЗАМ СЛОВА СВОЙСТВЕННЫ «ПЕРЕДАЧА ГЕРОЯМ 
ЖИЗНЕННОЙ ЭНЕРГИИ КАК ЗВУЧАНИЕМ, ТАК И КОЛЕБАНИЕМ» (ПУШКАРЕВА 2002, С. $28-38 ; 2007$, с. $35-36)$. ГОВОРИТЬ И СЛОВОМ МОЖНО РАЗРУШИТЬ И САМ СВЯЩЕННЫЙ ОБЪЕКТ, И СВОЙ КОНТАКТ С НИМ. ТАКИЕ НОРМЫ ОТНОСЯТСЯ В ПЕРВУЮ ОЧЕРЕДЬ К САКРАЛЬНОЙ СФЕРЕ. МНОГИЕ АКТИВИСТЫ И УЧЕНЫЕ ИЗ ЧИСЛА АБОРИГЕНОВ СЧИТАЮТ, ЧТО НЕЛЬЗЯ ДАЖЕ ЗАДАВАТЬ ВОПРОСЫ О ТАКИХ ВЕЩАХ.

СВЯТЫЕ СКАЗАНИЯ - ЭТО ТАБУ. ОНИ РАССКАЗЫВАЮТСЯ ТОЛЬКО В КОНКРЕТНЫХ СЛУЧАЯХ ОПРЕДЕЛЕННЫМ ЧЕЛОВЕКОМ ДОВЕРЕННОМУ ЛИЦУ, КОТОРЫЙ ПРИ ЭТОМ ПОЛУЧАЕТ ТОЧНОЕ УКАЗАНИЕ О ДАЛЬНЕЙШИХ ДЕЙСТВИЯХ В ОТНОШЕНИИ ПОЛУЧЕННОЙ ИНФОРМАЦИИ. ЭТО НАДО ВСЕГДА ИМЕТЬ В ВИДУ. САМЫЙ ВЕРНЫЙ ПУТЬ ЗАКРЫТЬ ПЕРЕД СОБОЙ ДВЕРИ ДОВЕРИЯ ОБСКИХ УГРОВ, ЭТО СПРОСИТЬ ИХ О «СВЯТЫХ СКАЗАНИЯХ» (ПЕСИКОВА 2000, С. 133). ИНФОРМАНТОВ-АБОРИГЕНОВ О МОЛЧАНИИ, Я ДАЖЕ УСЛЫШАЛА В СВОЙ АДРЕС: «А ТЫ КОЕ-ЧТО СТАЛА ПОНИМАТЬ». В ИХ КУЛЬТУРЕ МОЛЧАНИЕ - КОД.

О НЕКОТОРЫХ ВЕЩАХ НЕЛЬЗЯ ГОВОРИТЬ ЖЕНЩИНАМ ИЛИ НЕЛЬЗЯ ГОВОРИТЬ В КАКИХ ТО СИТУАЦИЯХ, НАПРИМЕР, ПЕРЕД ОХОТОЙ ИЛИ ВЕЧЕРОМ. ЕЩЕ БОЛЕЕ СТРОГИЕ НОРМЫ СУЩЕСТВУЮТ В ОТНОШЕНИИ ПОВЕДЕНИЯ В ЛЕСУ, ТАМ НЕЛЬЗЯ НАРУШАТЬ ТИШИНУ ПОКОЙ ДУХОВ. МОЛЧАНИЕ ИГРАЕТ БОЛЬШУЮ РОЛЬ И В ИХ ПОВСЕДНЕВНОЙ ЖИЗНИ АБОРИГЕНОВ, СУЩЕСТВУЕТ МНОЖЕСТВО ПРАВИЛ, ОСОБЕННО В ОТНОШЕНИИ НАИБОЛЕЕ ВАЖНЫХ СТОРОН ЖИЗНИ. НАПРИМЕР, НЕЛЬЗЯ ГОВОРИТЬ О ДЕТЯХ ДО ГОДА, ТАК КАК ТАКИЕ ДЕТИ НАХОДЯТСЯ НА ПОГРАНИЧЬЕ МИРОВ ДУХОВ И ЛЮДЕЙ. НЕЛЬЗЯ ГОВОРИТЬ И О РЕЗУЛЬТАТАХ ПРОМЫСЛА. МНОГИЕ ИНФОРМАНТЫ РАССКАЗЫВАЛИ, ЧТО ОНИ С ДЕТСКИХ ЛЕТ УСВОИЛИ НОРМУ: КОГДА ОХОТНИК ПРИШЕЛ ИЗ ЛЕСА, ЕГО НЕ НУЖНО НИ О ЧЕМ СПРАШИВАТЬ (СМ. ТАКЖЕ: ЛАПИНА 1998 С. 29). О ПРОМЫСЛЕ ВООБЩЕ ЛУЧШЕ НЕ ГОВОРИТЬ, ТАК КАК, ПО ПРЕДСТАВЛЕНИЯМ ОХОТНИКОВ, ЗВЕРИ МОГУТ УСЛЫШАТЬ. СУЩЕСТВУЕТ ПРАВИЛО, ПО КОТОРОМУ НЕЛЬЗЯ ГОВОРИТЬ О СВОИХ ВОЗМОЖНЫХ УСПЕХАХ В БУДУЩЕМ. О ПОСЛЕДНЕМ ДАЖЕ СУЩЕСТВУЕТ СЦЕНКА НА МЕДВЕЖЬЕМ ПРАЗДНИКЕ. В 1981 Г. Я НАБЛЮДАЛА ТАКОЕ ПРЕДСТАВЛЕНИЕ В Д. В. НИЛЬДИНО В ХМАО. СЦЕНКА СОПРОВОЖДАЛАСЬ ПЕСНЕЙ И ИГРОЙ НА МУЗЫКАЛЬНОМ СТРУННОМ ИНСТРУМЕНТЕ - САНГУЛЬТАПЕ. ВОТ ЕЕ СОДЕРЖАНИЕ:

«СТАРЫЙ ЛОСЬ НОЧЬЮ СПИТ, СЛЫШИТ ВО СНЕ: «ЗАВТРА В ПОЛДЕНЬ ПРИДУТ ОХОТНИКИ, БУДУТ ВАС ГНАТЬ». ОН ГОВОРИТ МОЛОДОМУ ЛОСЕНКУ: «ТЕБЯ ОХОТНИКИ УБЬЮТ, А Я УЙДУ ЗА УРАЛ». УТРОМ ПОБЕЖАЛИ ОЛЕНИ. МОЛОДОЙ ЛОСЬ СЛЫШИТ, ОХОТНИКИ РЯДОМ, ВОТ-ВОТ ДОГОНЯТ. ЛОСЕНОК ЛЕГКИЙ, ОН ПОБЕЖАЛ ВПЕРЕД БЫСТРЕЕ, ВСЕХ ОБОГНАЛ И УБЕЖАЛ. ВДРУГ СЛЫШИТ ВЫСТРЕЛ, ЭТО СТАРОГО ЛОСЯ ВОЖАКА УБИЛИ».

ОБЪЯСНЯЯ СОДЕРЖАНИЕ ПЕСНИ, ИСПОЛНИТЕЛЬ СКАЗАЛ, ЧТО ЭТО СЛУЧИЛОСЬ ПОТОМУ, ЧТО СТАРЫЙ ЛОСЬ СКАЗАЛ ЗАРАНЕЕ: «НИКОГДА НЕЛЬЗЯ ЗАРАНЕЕ ГОВОРИТЬ, ОСОБЕННО ПЕРЕД ОХОТОЙ». ЗДЕСЬ ОХОТНИЧЬИ ТАБУ ПЕРЕНОСЯТСЯ НА МИР ЖИВОТНЫХ (НОВИКОВА 1995, С. 145). МЕДВЕЖИЙ ПРАЗДНИК ОБСКИХ УГРОВ БЫЛ НЕ ТОЛЬКО ГЛАВНЫМ ПРАЗДНИКОМ, СОБРАНИЕМ ЖИЗНЕННО ВАЖНЫХ ОБРЯДОВ, ОН ВЫПОЛНЯЕТ ФУНКЦИЮ ПЕРЕДАЧИ ТРАДИЦИЙ, ЗНАНИЙ, НОРМ СЛЕДУЮЩИМ ПОКОЛЕНИЯМ. НЕОБХОДИМОСТЬ МОЛЧАНИЯ В БЫТУ ДИКТУЕТСЯ ЖИЗНЕННЫМИ УСЛОВИЯМИ ПРОЖИВАНИЕ НА СТОЙБИЩЕ ИЛИ В ЧУМЕ МНОГИХ ЛЮДЕЙ ЕСТЕСТВЕННО ВЫЗЫВАЕТ 
ТАКИЕ ТРЕБОВАНИЯ. НО ДЛЯ АБОРИГЕНОВ ЕСТЬ РАЗЛИЧИЕ МЕЖДУ МОЛЧАНИЕМ КАК СКРЫВАНИЕМ ВАЖНОЙ ИНФОРМАЦИИ И БЫТОВЫМ МОЛЧАНИЕМ, С КОТОРЫМ СТАЛКИВАЮТСЯ МНОГИЕ ПРИЕЗЖИЕ, ВПЕРВЫЕ ПОПАВШИЕ НА СТОЙБИЩЕ. ПОКАЗАТЕЛЬНОЙ ЯВЛЯЕТСЯ ПОГОВОРКА «ДЕТЕЙ И ОЛЕНЕЙ НЕ СЧИТАЮТ». НЕ НУЖНО СПРАШИВАТЬ, СКОЛЬКО У СЕМЬИ ОЛЕНЕЙ - НЕ НУЖНО СТАВИТЬ ЛЮДЕЙ В СИТУАЦИЮ НАРУШЕНИЯ НОРМЫ. МОЛЧАНИЕ, ТАКИМ ОБРАЗОМ, В ОБЖИТОМ, ОСВОЕННОМ ПРОСТРАНСТВЕ РЕГУЛИРУЕТСЯ ОПРЕДЕЛЕННЫМИ ПРАВИЛАМИ, ПРИЧЕМ ОНИ ЗАВИСЯТ НЕ ТОЛЬКО ОТ ПРОСТРАНСТВА, НО И ВРЕМЕНИ - ОСОБЕННО МНОГО ЗАПРЕТОВ СУЩЕСТВУЕТ ДЛЯ ВЕЧЕРА.

НА СТОЙБИЩАХ НЕ СПЕШАТ РАССКАЗАТЬ ВСЕ, ЧТО ЗНАЮТ И ВИДЕЛИ. ИНОГДА ЧЕЛОВЕК, ВЕРНУВШИСЬ С ОЛЕНЯМИ К ДОМУ, СКАЖЕТ ПАРУ СЛОВ, А ЕГО ДОМОЧАДЦЫ УЖЕ ВСЕ ЗНАЮТ, КАКОЙ ДОРОГОЙ ОНИ ШЛИ, ГДЕ ОСТАНАВЛИВАЛИСЬ НОЧЕВАТЬ, ЧТО ВИДЕЛИ ПО ДОРОГЕ. А.С. ПЕСИКОВА НАЗЫВАЕТ ЭТУ ЧЕРТУ ИХ КУЛЬТУРЫ ЗАКРЫТАЯ ОТКРЫТОСТЬ. ОНА ПИШЕТ:

В СРЕДЕ ПИМСКИХ ХАНТЫ ВЫ ЧАЩЕ ВСТРЕТИТЕ ЛЮДЕЙ, ШИРОКО ОТКРЫТЫХ, ДО НАИВНОСТИ. НО ЭТА ОТКРЫТОСТЬ КАЖУЩАЯСЯ. ИСТОРИЧЕСКИЙ ОПЫТ СОХРАНЕНИЯ СВОЕГО ЭТНОСА ПОДСКАЗАЛ ОБСКИМ УГРАМ: ВСЕ ЗАВЕТНОЕ ХРАНИТЬ ПОДАЛЬШЕ ОТ ЧУЖОГО, ОТ НЕДОБРОГО. ПИМСКИЕ ХАНТЫ ВСЕГДА ГОСТЕПРИИМНЫ: И НАКОРМЯТ, И СПАТЬ УЛОЖАТ, НО НЕ ВСЕ БУДУТ РАССКАЗЫВАТЬ, НЕ ВСЕ ПОКАЗЫВАТЬ. (ПЕСИКОВА 1996, с. 230)

\section{Молчание как протест}

В СОВРЕМЕННЫХ УСЛОВИЯХ МОЛЧАНИЕ ПРОЯВЛЯЕТСЯ И КАК ФОРМА СОЦИАЛЬНОГО ПРОТЕСТА. НАПРИМЕР, В РАЙОНАХ МЕСТОРОЖДЕНИЙ, КОТОРЫЕ НАХОДЯТСЯ ПРЯМО ЗА РОДОВЫХ УГОДЬЯХ ХАНТОВ, МНЕ ПРИХОДИЛОСЬ ВСТРЕЧАТЬ ПОДРОСТКОВ И ДАЖЕ МОЛОДЫХ ЛЮДЕЙ, КОТОРЫЕ ВООБЩЕ НЕ ЗНАЮТ РУССКОГО ЯЗЫКА. К СОЖАЛЕНИЮ, МНОГИЕ ХАНТЫ В ТАКИХ МЕСТАХ НЕ ВИДЯТ ДЛЯ СЕБЯ БУДУЩЕГО И СЧИТАЮТ, ЧТО ДЕТЕЙ НЕ НУЖНО ОТДАВАТЬ В ИНТЕРНАТ, ТАМ ИМ БУДЕТ ПЛОХО, А НАСТОЯЩЕГО ОБРАЗОВАНИЯ ОНИ ВСЕ РАВНО НЕ ПОЛУЧАТ. Я СТАЛКИВАЛАСЬ В ТАКОЙ СИТУАЦИЕЙ В РАЙОНЕ ТЯНСКОГО МЕСТОРОЖДЕНИЯ ВО ВТОРОЙ ПОЛОВИНЕ 1990-Х ГОДОВ.

ИНОГДА ПРОТЕСТ ПРОЯВЛЯЕТСЯ В ВИДЕ МОЛЧАНИЯ ДЕТЕЙ СО СТОЙБИЩ В ИНТЕРНАТЕ ИЛИ ШКОЛЕ. НА ОСНОВАНИИ ЭТОГО, ТАКИХ ДЕТЕЙ ДАЖЕ ИНОГДА ОТПРАВЛЯЮТ В ШКОЛЫ ДЛЯ СЛАБОРАЗВИТЫХ, ВМЕСТО ТОГО, ЧТОБЫ РАБОТАТЬ С НИМИ БОЛЕЕ ВНИМАТЕЛЬНО И ПРОФЕССИОНАЛЬНО. КОГДА Я РАЗГОВАРИВАЛА СО СТУДЕНТАМИ, ОБУЧАЮЩИМИСЯ В ЮГОРСКОМ УНИВЕРСИТЕТЕ В Г. ХАНТЫ-МАНСИЙСКЕ, МНОГИЕ ИЗ НИХ ГОВОРИЛИ, ЧТО САМЫМ СИЛЬНЫМ НЕГАТИВНЫМ ВОСПОМИНАНИЕМ О ПЕРВЫХ ДНЯХ В ШКОЛЕ БЫЛО ТО, ЧТО «УЧИТЕЛЬНИЦА КРИЧАЛА».

МОЛЧАНИЕ ИГРАЕТ ВАЖНУЮ РОЛЬ В КОММУНИКАТИВНОЙ АДАПТАЦИИ. ТАК, НАПРИМЕР, АППАЧИ МОЛЧАТ В СИТУАЦИЯХ, КОГДА КТО-ТО БЫВАЕТ В ГНЕВЕ, БЕШЕНСТВЕ. В ЭТОЙ СИТУАЦИИ ТРАДИЦИЯ ПРЕДПИСЫВАЕТ СОХРАНЯТЬ МОЛЧАНИЕ (Basso, 1970, p. 213-230). АБОРИГЕНЫ РОССИЙСКОГО СЕВЕРА ТАКЖЕ ИСПОЛЬЗУЮТ ТАКУЮ ТАКТИКУ ПОВЕДЕНИЯ С НЕФТЯНИКАМИ. ВО ВРЕМЯ ПОДПИСАНИЯ ЭКОНОМИЧЕСКИХ СОГЛАШЕНИЙ КОМПАНИИ УСТРАИВАЮТ СОБРАНИЯ, НА КОТОРЫХ ОНИ СОБИРАЮТ ВСЕХ ХАНТОВ И НЕНЦЕВ СО СТОЙБИЩ И ОБЪЯСНЯЮТ ИМ УСЛОВИЯ ЗАКЛЮЧЕНИЯ СОГЛАШЕНИЙ И ИХ УСЛОВИЯ. ВО ВТОРОЙ ПОЛОВИНЕ 1990-Х НА ТАКИХ 
СОБРАНИЯХ ЧАСТО ВЫЯСНЯЛОСЬ, ЧТО НЕ ВСЕ УСЛОВИЯ ВЫПОЛНЕНЫ, НО АБОРИГЕНЫ МОЛЧАЛИ. НО ЭТО БЫЛО НЕ МОЛЧАНИЕ - ПОРАЖЕНИЕ, КАК МОЖЕТ ПОКАЗАТЬСЯ НА ПЕРВЫЙ ВЗГЛЯД. ОНИ МОЛЧАЛИ И ВЫЖИДАЛИ, ОТСТУПАЛИ, ТАК КАК ДУМАЛИ, ЧТО НЕФТЯНИКИ УЙДУТ ИЛИ СЧИТАЛИ, ЧТО СМОГУТ ПОЛУЧИТЬ ОТ НЕФТЯНИКОВ ОБЕЩАННОЕ, ТАК КАК «ПОСМОТРЕЛИ В ГЛАЗА ДРУГ ДРУГУ», А ЭТОГО ПО НОРМАМ ИХ ОБЫЧНОГО ПРАВА, ДОСТАТОЧНО. О ПОДОБНОМ ВЫЖИДАТЕЛЬНОМ МОЛЧАНИИ ПИШЕТ И ИССЛЕДОВАТЕЛЬ ИСЛАНДСКИХ САГ Э. ЭСТЕРБЕРГ. ОНА ОТМЕЧАЕТ, ЧТО В ПОДОБНЫХ СИТУАЦИЯХ ЛЮДИ В САГАХ ХРАНЯТ МОЛЧАНИЕ, ПОСКОЛЬКУ СЛОВО МОЖЕТ ТОЛЬКО УСУГУБИТЬ ВОЗНИКШУЮ СИТУАЦИЮ ПРОТИВОСТОЯНИЯ. МОЛЧАНИЕ ПРЕДСТАВЛЯЕТ СОБОЙ ПРОМЕЖУТОЧНУЮ СТУПЕНЬ НА ПУТИ ОТ ОДНОГО ДЕЙСТВИЯ К ДРУГОМУ, НО ОТНЮДЬ НЕ КОНЕЧНЫЙ ЭТАП. ТАКОЕ НАСЫЩЕННОЕ ВЫЖИДАТЕЛЬНОЕ МОЛЧАНИЕ АВТОР ВЫВОДИТ ИЗ МОДЕЛИ «ОДНОУКЛАДНОЙ» КУЛЬТУРЫ (ЭСТЕРБЕРГ 1996, с. 35). ДРУГУЮ ТРАКТОВКУ МОЛЧАНИЯ И СЛОВА В САГАХ ДАЕТ А.Я. ГУРЕВИЧ, СЧИТАЯ ИХ ВОЗМОЖНЫМИ ИСТОКАМИ И РЕГУЛЯТОРАМИ СУДЕБНОЙ МЕНТАЛЬНОСТИ, ТАК КАК В САГАХ СЮЖЕТЫ, СВЯЗАННЫЕ С СУДЕБНЫМИ РАЗБИРАТЕЛЬСТВАМИ ДОСТАТОЧНО РАСПРОСТРАНЕНЫ. А ЧЕЛОВЕК СМОТРИТ НА СЕБЯ КАК БЫ СО СТОРОНЫ, ТАК КАК ЗАВИСИТ ОТ СУЖДЕНИЯ СОЦИАЛЬНОЙ ГРУППЫ (ГУРЕВИЧ 1996, С. 43-46). У РОССИЙСКИХ АБОРИГЕНОВ МОЛЧАНИЕ В ОТНОШЕНИЯХ С НЕФТЯНИКАМИ ЯВЛЯЕТСЯ ИХ ПОПЫТКОЙ УСТАНОВИТЬ КОНТАКТ ЧЕРЕЗ МОЛЧАНИЕ, ДАТЬ ВОЗМОЖНОСТЬ СОБЕСЕДНИКУ ПОЧУВСТВОВАТЬ СОПРИЧАСТНОСТЬ. СЛОЖНОСТЬ И ДАЖЕ ОПАСНОСТЬ ТАКОЙ ТАКТИКИ ЗАКЛЮЧАЕТСЯ В ТОМ, ЧТО НЕФТЯНИКИ (КАК ВПРОЧЕМ, ЧИНОВНИКИ И Т.Д.) ВОСПРИНИМАЮТ КОММУНИКАЦИЮ БОЛЕЕ ОДНОЗНАЧНО.

В КУЛЬТУРЕ АБОРИГЕНОВ ЕСТЬ СЕМАНТИЧЕСКИ НАСЫЩЕННЫЕ СИМВОЛЫ, ИСПОЛЬЗУЯ КОТОРЫЕ ОНИ МОГУТ ПЕРЕДАТЬ ИНФОРМАЦИЮ НЕ ТОЛЬКО ВНУТРИ СООБЩЕСТВА, НО И ВОВНЕ. ВОЗМОЖНО, ДАЖЕ САМИ АБОРИГЕНЫ НЕ ЗАДУМЫВАЮТСЯ ЧАСТО НАД ТЕМ, ПОЧЕМУ ОНИ ИСПОЛЬЗУЮТ ТЕ ИЛИ ИНЫЕ ЭЛЕМЕНТЫ СВОЕЙ КУЛЬТУРЫ СЕГОДНЯ. ЭТО ПРОИСХОДИТ КАК БЫ ЕСТЕСТВЕННО, ТО ЕСТЬ, ВКЛЮЧАЯ ПОЛИТИЧЕСКУЮ БОРЬБУ В СВОЮ ЖИЗНЬ, А ЗНАЧИТ И КУЛЬТУРУ, ОНИ ПРИДАЮТ ЕЙ ТЕ ФОРМЫ, КОТОРЫЕ ЗНАЧИМЫ ДЛЯ ИХ ОБРАЗА ЖИЗНИ. В КУЛЬТУРЕ АБОРИГЕНОВ БОЛЬШЕ ИСПОЛЬЗУЕТСЯ ДЕЙСТВИЕ, ЧЕМ СЛОВО. НАПРИМЕР, ДЛЯ ТОГО, ЧТОБЫ ВЫСКАЗАТЬ СВОИ ПРЕТЕНЗИИ К ПРОМЫШЛЕННЫМ КОМПАНИЯМ, АБОРИГЕНЫ В 1990-Х ГГ. БЕЗ ЛИШНИХ СЛОВ МОГЛИ ПОСТАВИТЬ ЧУМ В ГОРОДЕ. ВО ВРЕМЯ ОДНОГО ИЗ ПИКЕТОВ, ОРГАНИЗОВАННОГО ПОЭТОМ-ОЛЕНЕВОДОМ ЮРИЕМ ВЭЛЛОЙ, В ХАНТЫ-МАНСИЙСКОМ АВТОНОМНОМ ОКРУГЕ В НАЧАЛЕ 1990-ХГГ. ОСОБЕННО ЯРКО ПРОЯВИЛАСЬ РИТУАЛИЗАЦИЯ СОВРЕМЕННОЙ ПОЛИТИЧЕСКОЙ КУЛЬТУРЫ НАРОДОВ СЕВЕРА. У НЕНЦЕВ БЫЛ ТАКОЙ ОБРЯД - ЕСЛИ В ЧУМЕ УМИРАЛ ЧЕЛОВЕК, ЕГО МОГЛИ ТАМ ОСТАВИТЬ, ПОВЕРНУВ ЧУМ ВХОДОМ НА "НОЧНУЮ СТОРОНУ". ЧУМ ПРЕВРАЩАЛСЯ В МОГИЛУ. И Ю.ВЭЛЛА РЕШИЛ ВОСПРОИЗВЕСТИ ЭТОТ ОБРЯД ВО ВРЕМЯ ПИКЕТА.

ЧУМ ПОСТАВИЛИ ПЕРЕД ЗДАНИЕМ АДМИНИСТРАЦИИ. В НЕМ НАХОДИЛОСЬ ЧУЧЕЛО, ИЗОБРАЖАВШЕЕ АДМИНИСТРАЦИЮ. НА ЧУМЕ НАДПИСЬ - "ОСТОРОЖНО! В ЧУМЕ БОЛЬНОЙ. НЕ КРИЧАТЬ!" НА ЧУЧЕЛЕ ЛИСТОВКА: "ПРИБЛИЗИТЕЛЬНЫЙ ДИАГНОЗ НЕФТЯНАЯ ЧУМА? ОКОНЧАТЕЛЬНЫЙ ДИАГНОЗ - ..." ПОСЛЕДНЕЕ ДОЛЖНА БЫЛА ОПРЕДЕЛИТЬ АДМИНИСТРАЦИЯ ОКРУГА (ГЛУХИХ 1995, ПРА). КОГДА ЕЩЕ ДО ПИКЕТА Ю. ВЭЛЛА РАССКАЗЫВАЛ О СВОИХ ПЛАНАХ, ОН СКАЗАЛ:

ЕСЛИ АДМИНИСТРАЦИЯ БУДЕТ С НАМИ ГОВОРИТЬ И СОГЛАСИТСЯ НЕ ТРОГАТЬ НАШУ ЗЕМЛЮ, МЫ ПОЙМЕМ, ЧТО АДМИНИСТРАЦИЯ ВЫЛЕЧИЛАСЬ, И РАЗБЕРЕМ ЭТОТ ЧУМ. 
ЕСЛИ НЕТ, МЫ БУДЕМ СЧИТАТЬ, ЧТО БОЛЬНОЙ УМЕР, И ЧУМ ОСТАВИМ ПРЯМО НА ПЛОЩАДИ, ПОВЕРНУВ ЕГО НА НОЧНУЮ СТОРОНУ. МЫ КОРЕННЫЕ ЖИТЕЛИ БУДЕМ СЧИТАТЬ, ЧТО НАША АДМИНИСТРАЦИЯ ЗАРАЗИЛАСЬ ТЯЖЕЛОЙ БОЛЕЗНЬЮ И УМЕРЛА. МЕСТОРОЖДЕНИЙ. ЯРКАЯ ТЕАТРАЛЬНОСТЬ ПИКЕТА НЕ ТОЛЬКО ПРИВЛЕКЛА К НЕМУ ВНИМАНИЕ, НО И СВИДЕТЕЛЬСТВУЕТ О ПОИСКАХ КОМПРОМИССА С ВЛАСТЬЮ И НЕФТЯНИКАМИ В ПЕРВУЮ ОЧЕРЕДЬ СО СТОРОНЫ АБОРИГЕНОВ. ВЕДЬ ЦЕЛЬ ЭТОЙ БОРЬБЫ - НЕ ВЕРНУТЬ ЗЕМЛИ И ПРЕКРАТИТЬ НЕФТЕДОБЫЧУ, КАК МОЖЕТ НА ПЕРВЫЙ ВЗГЛЯД ПОКАЗАТЬСЯ, А ОСТАНОВИТЬ ЕЕ НА ТЕХ ГРАНИЦАХ, КОТОРЫЕ СЕГОДНЯ СУЩЕСТВУЮТ МЕЖДУ ПРОМЫШЛЕННО ОСВОЕННЫМИ ТЕРРИТОРИЯМИ И ЗЕМЛЯМИ ТРАДИЦИОННОГО ПРИРОДОПОЛЬЗОВАНИЯ. ПОЛИТИЧЕСКАЯ БОРЬБА АБОРИГЕНОВ ОБЛЕКАЕТСЯ В ФОРМУ ПИКЕТОВ, НО ОНА НЕ АГРЕССИВНА. ОНИ НЕ НАХОДЯТ ОТКЛИКА, ИХ НЕ ПОНИМАЮТ И НЕ ПРИНИМАЮТ, А ОНИ СНОВА И СНОВА ВЫХОДЯТ НА ПЛОЩАДЬ.

ВАЖНЫМИ ЭЛЕМЕНТАМИ СЛОЖИВШЕЙСЯ СИНКРЕТИЧНОЙ ПОЛИТИЧЕСКОЙ КУЛЬТУРЫ КОРЕННЫХ НАРОДОВ СЕВЕРА СТАЛА ПРЕЗЕНТАЦИЯ "СВОЕЙ" КУЛЬТУРЫ ЧЕРЕЗ ОБРАЗЫ КУЛЬТУРЫ "ПРИШЛОГО" НАСЕЛЕНИЯ И ВКЛЮЧЕНИЕ ПОЛИТИЧЕСКОЙ СИСТЕМЫ СТРАНЫ В КАРТИНУ МИРА АБОРИГЕНОВ. ПРОЦЕСС ЭТОТ ПРОИСХОДИТ НА НАШИХ ГЛАЗАХ, ЧТО СОЗДАЕТ ОПРЕДЕЛЕННЫЕ ТРУДНОСТИ ПРИ ЕГО ИНТЕРПРЕТАЦИИ. ПРИЧЕМ ПРИ ИССЛЕДОВАНИИ ПОЛИТИЧЕСКОЙ КУЛЬТУРЫ МЫ ИМЕЕМ ДЕЛО СКОРЕЕ С ИНТЕРПРЕТАЦИЯМИ ИНТЕРПРЕТАЦИЙ И ЩУГАДЫВАНИЕ ЗНАЧЕНИЙ, ОЦЕНИВАНИЕ ДОГАДОК И ВЫВЕДЕНИЕ ПОЯСНЯЮЩИХ ЗАКЛЮЧЕНИЙ ИЗ НАИБОЛЕЕ УДАЧНЫХ ДОГАДОК», ПО СЛОВАМ К. ГИРЦА (ГИРЦ 2004, С. 28), СТРОИТСЯ В ДАННОЙ РАБОТЕ НА СООТНЕСЕНИИ ДЕКЛАРАЦИЙ И ДЕЙСТВИЙ КАК СО СТОРОНЫ АБОРИГЕНОВ, ТАК И СО СТОРОНЫ ЧАСТО ПРОТИВОСТОЯЩЕГО ИМ МИРА ГОСУДАРСТВЕННОЙ ВЛАСТИ И ЧАСТНОГО БИЗНЕСА. И ЕСЛИ МЕХАНИЗМЫ УСТАНОВЛЕНИЯ ДИАЛОГА МЕЖДУ ГОСУДАРСТВОМ И ОБЩЕСТВОМ, ЧАСТЬЮ КОТОРОГО ЯВЛЯЮТСЯ АБОРИГЕНЫ, НАМ БОЛЕЕ ИЗВЕСТНЫ, ТО АБОРИГЕННАЯ ПОЛИТИЧЕСКАЯ КУЛЬТУРА МАЛО ИССЛЕДОВАНА. ЭТА КУЛЬТУРА РАССМАТРИВАЕТСЯ КАК «ВЫРАЖАЮЩЕЕ ДЕЙСТВИЕ», ВКЛЮЧАЮЩЕЕ СТАНДАРТИЗОВАННЫЕ И УНИКАЛЬНЫЕ СИМВОЛЫ (ЛИЧ, 2001 с 18-19).

ОБРАЩЕНИЕ К РИТУАЛУ И РИТУАЛИЗИРОВАННОМУ ПОВЕДЕНИЮ В УСЛОВИЯХ КОНФЛИКТОВ В СФЕРЕ ПРИРОДОПОЛЬЗОВАНИЯ, СВЯЗАНО СО СТРЕМЛЕНИЕМ АБОРИГЕНОВ БЫТЬ УСЛЫШАННЫМИ, ДОНЕСТИ ДО ВЛАСТИ СВОИ ТРЕБОВАНИЯ В УСЛОВИЯХ, КОГДА ОБЫЧНЫЕ ПРАВОВЫЕ МЕТОДЫ ОКАЗЫВАЮТСЯ МАЛОЭФФЕКТИВНЫМИ. ЧЕРЕЗ РИТУАЛ ДЕЛАЕТСЯ ПОПЫТКА ВКЛЮЧИТЬ В СВОЙ МИР «НЕ ТРАДИЦИОННЫХ» УЧАСТНИКОВ (ПРЕЗИДЕНТА, АДМИНИСТРАЦИЮ).

ЧУМ НА ГОРОДСКОЙ ПЛОЩАДИ СТАНОВИТСЯ МИНИМУМОМ РИТУАЛА. СЕЙЧАС ДАЛЕКО НЕ ВСЕ АБОРИГЕНЫ ЖИВУТ В ЧУМАХ И ДАЖЕ НЕ ВСЕ ИМЕЮТ ЧУМЫ В КАЧЕСТВЕ ВРЕМЕННОГО ЖИЛИЩА. НО ЧУМ ИГРАЛ БОЛЬШУЮ РОЛЬ В ТРАДИЦИОННОЙ ОЛЕНЕВОДЧЕСКОЙ КУЛЬТУРЕ И ОН, НАРЯДУ С ОЛЕНЕМ, ЯВЛЯЕТСЯ ОБРАЗОМ ЭТОЙ КУЛЬТУРЫ. ЗДЕСЬ ЗНАЧИМО ВСЕ. ЧУМ КАК ЖИЛИЩЕ ОТРАЖАЕТ ОБРАЗ ЖИЗНИ. ОН НЕ ТОЛЬКО МАКСИМАЛЬНО АДАПТИРОВАН К ПРИРОДНЫМ УСЛОВИЯМ, НО ПО НЕМУ МОЖНО ПРОСЛЕДИТЬ И СОЦИАЛЬНЫЙ СТАТУС ЖИВУЩИХ В НЕМ ЛЮДЕЙ, И ИХ МИФОЛОГИЧЕСКИЕ ПРЕДСТАВЛЕНИЯ, А ПРИ БОЛЕЕ ГЛУБОКОМ ИССЛЕДОВАНИИ И В ЦЕЛОМ СУЩЕСТВУЮЩУЮ В ЭТОМ ОБЩЕСТВЕ МОДЕЛЬ МИРА. ЧУМ В ГОРОДЕ И ПО ЕГО ФОРМЕ, И ПО МАТЕРИАЛУ, ИЗ КОТОРОГО СДЕЛАН (ОЛЕНЬИ ШКУРЫ), ВОСПРИНИМАЕТСЯ

Études finno-ougriennes, 47 | 2015 
КАК ЗНАК АБОРИГЕННОЙ, ЧУЖОЙ, АЛЬТЕРНАТИВНОЙ КУЛЬТУРЫ ДАЖЕ ДЛЯ ТЕХ ЛЮДЕЙ, КОТОРЫЕ НЕ БУДУТ ВДАВАТЬСЯ В РАЗЛИЧНЫЕ СЕМИОТИЧЕСКИЕ ТОНКОСТИ. ВОЗМОЖНО, ДАЖЕ САМИ АБОРИГЕНЫ НЕ ЗАДУМЫВАЮТСЯ ЧАСТО НАД ТЕМ, ПОЧЕМУ ОНИ ИСПОЛЬЗУЮТ ТЕ ИЛИ ИНЫЕ ЭЛЕМЕНТЫ СВОЕЙ КУЛЬТУРЫ. ЭТО ПРОИСХОДИТ КАК БЫ ЕСТЕСТВЕННО, ТО ЕСТЬ, ВКЛЮЧАЯ ПОЛИТИЧЕСКУЮ БОРЬБУ В СВОЮ ЖИЗНЬ, А ЗНАЧИТ И КУЛЬТУРУ, ОНИ ПРИДАЮТ ЕЙ ТЕ ФОРМЫ, КОТОРЫЕ ЗНАЧИМЫ ДЛЯ ИХ ОБРАЗА ЖИЗНИ. ПОЭТОМУ САМА ФОРМА ПИКЕТА, ВЫБРАННАЯ Ю. ВЭЛЛОЙ, ОЧЕНЬ ОРГАНИЧНА ДЛЯ ИХ КУЛЬТУРЫ. В ЭТОМ СЛУЧАЕ ЧУМ ВЫСТУПАЕТ НЕ ТОЛЬКО ЗНАКОМ КОНФРОНТАЦИИ, НО И ПОПЫТКОЙ УСТАНОВЛЕНИЯ ДИАЛОГА, ЧЕРЕЗ ЧУМ НА ПЛОЩАДИ ПРОИСХОДИТ ОСВОЕНИЕ НОВОГО ДЛЯ АБОРИГЕНОВ ПРОСТРАНСТВА ГОРОДСКОЙ, ГОСУДАРСТВЕННОЙ кУльтУры.

ПОДОБНЫЙ ПОДХОД ПОДРОБНО ИССЛЕДОВАН В. ТЭРНЕРОМ НА ПРИМЕРЕ ОТНОШЕНИЯ К БЛИЗНЕЧЕСТВУ В АФРИКЕ. ОН ПИШЕТ:

В ОБЩЕСТВЕ, ОСНОВАННОМ НА РОДСТВЕ С БЛИЗНЕЧЕСТВОМ МОЖНО СДЕЛАТЬ ДВЕ ВЕЩИ. ЛИБО МОЖНО СКАЗАТЬ, ПОДОБНО МАЛЬЧИКУ, ВПЕРВЫЕ УВИДЕВШЕМУ ЖИРАФА: «Я НЕ ВЕРЮ ЭТОМУ» И ОТРИЦАТЬ СОЦИАЛЬНОЕ СУЩЕСТВОВАНИЕ БИОЛОГИЧЕСКОГО ФАКТА, ИЛИ ЖЕ, ПРИНИМАЯ ФАКТ, МОЖНО ПОПЫТАТЬСЯ УЧЕСТЬ ЕГО. ЕСЛИ ПОПЫТАТЬСЯ УЧЕСТЬ ФАКТ, ТО НУЖНО, ПОСКОЛЬКУ ЭТО ВОЗМОЖНО, ЗАСТАВИТЬ ЕГО КАЗАТЬСЯ НЕ ПРОТИВОРЕЧАЩИМ ВСЕЙ КУЛЬТУРЕ" (ТЭРНЕР 1983, С. 140). ДЛЯ РАЗРЕШЕНИЯ ВОЗНИКШЕГО КОНФЛИКТА В ТРАДИЦИОННОЙ КУЛЬТУРЕ ВОЗМОЖНЫ ДВЕ ТАКТИКИ РИТУАЛЬНОГО ПОВЕДЕНИЯ «ЧУЖОЕ ЛИБО ВЫПРОВАЖИВАЕТСЯ ИЗ СВОЕГО МИРА, ЛИБО ЕГО ПРЕВРАЩАЮТ В СВОЕ (БАЙБУРИН 1993, c.191).

ДЛЯ ХАНТОВ И ЛЕСНЫХ НЕНЦЕВ ЧУМ, КАК УКАЗЫВАЛОСЬ, КАК ВРЕМЕННОЕ ЖИЛИЩЕ СТАНОВИТСЯ И УБЕЖИЩЕМ ПРИ ВЫНУЖДЕННЫХ ПЕРЕМЕЩЕНИЯХ, КОГДА НЕФТЯНИКИ ЗАНИМАЮТ ВСЕ НОВЫЕ ЗЕМЛИ. ИЗ-ЗА НЕДОСТАТКА ПАСТБИЩ АБОРИГЕНЫ В ХМАО ВЫНУЖДЕНЫ СОКРАЩАТЬ СВОЕ КОЧЕВАНИЕ, ПРИ ЭТОМ Я ВИДЕЛА У ЛЕСНЫХ НЕНЦЕВ ИЗБУШКИ ВНУТРИ ОФОРМЛЕННЫЕ КАК ЧУМЫ. ВОЗМОЖНО СО ВРЕМЕНЕМ У ЧУМА ПОЯВЯТСЯ ЕЩЕ КАКИЕ-ТО ФУНКЦИИ, НО КАК СРЕДСТВО И ПРОСТРАНСТВО БОРЬБЫ ОН ИСПОЛЬЗУЕТСЯ И АБОРИГЕНАМИ В ДРУГИХ СТРАНАХ, ТАКОЙ ОПЫТ ПРОШЛИ НОРВЕЖСКИЕ СААМИ.

\section{Молчание как код понимания}

АБОРИГЕНЫ СЕВЕРА МОГУТ ОБЩАТЬСЯ БЕЗ СЛОВ. ПОЛУЧИТЬ ОБЪЯСНЕНИЕ ЭТОМУ ФЕНОМЕНУ ТРУДНО, ОДНО ИЗ ВОЗМОЖНЫХ СОДЕРЖИТСЯ В РАБОТЕ А.С. ПЕСИКОВОЙ, КОТОРАЯ ПИШЕТ ОБ СУЩЕСТВОВАНИИ «ОРЕОЛА» ЛЮБОГО ПРЕДМЕТА, ОБЪЕКТА ИЛИ ЯВЛЕНИЯ, НА КОТОРОМ ЗАПЕЧАТЛЕВАЕТСЯ ВОЗДЕЙСТВИЕ ДРУГИХ ОБЪЕКТОВ (ПЕСИКОВА 2000, С. 132). ВОЗМОЖНО ИМЕННО ЭТО ПОЗВОЛЯЕТ ХАНТАМ ПЕРЕДАВАТЬ ИНФОРМАЦИЮ БЕЗ СЛОВ, СОВЕРШАЯ КАКИЕ-ТО ДЕЙСТВИЯ МЫСЛЕННО. ВЕДЬ О НЕКОТОРЫХ СВЯЩЕННЫХ МЕСТАХ НЕ ТОЛЬКО ГОВОРИТЬ НЕЛЬЗЯ, НА НИХ ДАЖЕ НЕЛЬЗЯ СМОТРЕТЬ. ПРИЧЕМ ЭТО ОТНОСИТСЯ И К ВПОЛНЕ БЫТОВЫМ ХОЗЯЙСТВЕННЫМ ПРАКТИКАМ, ТАКИМ КАК ОЛЕНЕВОДСТВО («МЫСЛЕННО ПАСТИ ОЛЕНЕЙ»).

ПОЧЕМУ ЖЕ СЕГОДНЯ АБОРИГЕНЫ НЕ ХОТЯТ ОТКРЫТЬ ВСЕ СВОИ ЗНАНИЯ ОБ ОКРУЖАЮЩЕМ МИРЕ И О СВЯЩЕННЫХ МЕСТАХ КАК ИХ ЧАСТИ. ВЕРОЯТНО, ЭТОМУ ИХ УЧИТ ПЕЧАЛЬНЫЙ ОПЫТ ПЕРВОНАЧАЛЬНОГО ПРОМЫШЛЕННОГО ОСВОЕНИЯ СЕВЕРА, 
КОГДА УЖЕ МНОГИЕ ПАМЯТНИКИ БЫЛИ РАЗРУШЕНЫ. СХОДНЫЙ ПУТЬ ПРОШЛИ И ДРУГИЕ КОРЕННЫЕ НАРОДЫ МИРА, В ЧАСТНОСТИ В США.

Я ОБСУЖДАЛА СУДЬБЫ РАЗВИТИЯ ТРАДИЦИОННЫХ КУЛЬТУР С АМЕРИКАНСКИМ ПИСАТЕЛЕМ Н. СКОТТОМ МОМАДЭЕМ, ВО ВРЕМЯ ЕГО ВИЗИТА В МОСКВУ В 2000 ГОДУ. ОН СКАЗАЛ, ЧТО

В ИНДЕЙСКОЙ ИСТОРИИ ЕСТЬ ПРИМЕРЫ, КОГДА ИХ ИДЕИ УХОДИЛИ ВОВНЕ, И ПРИХОДИЛО ЗЛО. ВОЗМОЖНО, ЭТО ОДНА ИЗ ПРИЧИН ТОГО, ЧТО НЕКОТОРЫЕ ПЛЕМЕНА НЕ ХОТЯТ ИМЕТЬ ПИСЬМЕННОСТЬ. ОН ПРИВЕЛ ПРИМЕР, КОГДА В ПЛЕМЕНАХ ЮГО-ЗАПАДА, УЧИТЕЛЬНИЦА НА УРОКЕ В ДЕРЕВНЕ НАПИСАЛА НА ДОСКЕ СЛОВО НА ЯЗЫКЕ ЭТОГО ПЛЕМЕНИ, ВОЖДЬ С НЕЙ БЕСЕДОВАЛ И ЗАПРЕТИЛ ПИСАТЬ ИХ СЛОВА. ОН СЧИТАЕТ, ЧТО ПОЭТОМУ И ПИСАТЕЛИ ИНДЕЙЦЫ ПИШУТ ПОАНГЛИЙСКИ, НЕТ ТРАДИЦИИ ПИСЬМЕННОЙ КУЛЬТУРЫ.

К. БАССО ОПИСЫВАЕТ ВИДЫ МОЛЧАНИЯ У АППАЧЕЙ, КОГДА ДЕТИ ВОЗВРАЩАЮТСЯ ИЗ ИНТЕРНАТА ДОМОЙ И РОДИТЕЛИ С НИМИ ПЕРВОЕ ВРЕМЯ НЕ РАЗГОВАРИВАЮТ. ЕГО ИНФОРМАНТЫ ТАК ОБЪЯСНЯЛИ ЭТУ СИТУАЦИЮ:

ДЕТИ В ШКОЛЕ ОБЩАЮТСЯ С БЕЛЫМИ ЛЮДЬМИ, НАХОДЯТСЯ ПОД ВЛИЯНИЕМ ИХ МЫСЛЕЙ И ЦЕННОСТЕЙ. ОНИ ЗАБЫВАЮТ, ОТКУДА ОНИ И СТЫДЯТСЯ ТОГО, ЧТО ИХ РОДИТЕЛИ БЕДНЫЕ. ОНИ ЗАБЫВАЮТ, ЧТО ОНИ АППАЧИ» ИЛИ «МЫ МОЛЧИМ С ДЕТЬМИ. ОНИ ДОЛГО НЕ БЫЛИ ДОМА, И МЫ НЕ ЗНАЕМ, КАК ИМ ТЕПЕРЬ НРАВИТСЯ БЫТЬ ДОМА. ЧЕРЕЗ КАКОЕ-ТО ВРЕМЯ, МЫ ВИДИМ, ЧТО ИМ НРАВИТСЯ ДОМА. ЭТО ЗНАЧИТ, ЧТО МЫ МОЖЕМ СЕБЯ ЧУВСТВОВАТЬ ХОРОШО. ЗНАЧИТ, МЫ СНОВА МОЖЕМ С НИМИ РАЗГОВАРИВАТЬ. ВСЕ СТАНОВИТСЯ, КАК БЫЛО ДО ИХ ОТЪЕЗДА» (BassO 1970, p. 213-230).

ЕГО ПРИМЕРЫ ПОКАЗЫВАЮТ, ЧТО ИНДЕЙЦЫ ПЕРЕЖИДАЮТ СИТУАЦИЮ НАРУШЕНИЯ ПОРЯДКА В МОЛЧАНИИ. ВЕРОЯТНО, МОЖНО СКАЗАТЬ, ЧТО ЛЮДИ ЧЕРЕЗ МОЛЧАНИЕ КАК ИНСТРУМЕНТ ПРОЖИВАЮТ, ПРОРАБАТЫВАЮТ ЭТУ СИТУАЦИЮ И ВОССТАНАВЛИВАЮТ порядок.

НЕСМОТРЯ НА ТО, ЧТО СОВРЕМЕННАЯ ПОЛИТИКА В ОТНОШЕНИИ КОРЕННЫХ НАРОДОВ СЕВЕРА СКЛАДЫВАЕТСЯ УЖЕ В ТЕЧЕНИЕ ДЕСЯТКОВ ЛЕТ, СОЗДАЕТСЯ ВПЕЧАТЛЕНИЕ, ЧТО ДЕЙСТВИТЕЛЬНЫЕ УСТРЕМЛЕНИЯ АБОРИГЕНОВ, ЗАИНТЕРЕСОВАННЫХ В СОХРАНЕНИИ И РАЗВИТИИ ТРАДИЦИОННОГО ПРИРОДОПОЛЬЗОВАНИЯ, ОСТАЮТСЯ НЕПОНЯТНЫМИ ВЛАСТИ. АБОРИГЕНЫ РАЗЛИЧНЫМИ СПОСОБАМИ ДЕМОНСТРИРУЮТ МИРУ ТОТ НЕПРОСТОЙ ФАКТ, ЧТО НА СЕВЕРЕ ПРЕДСТАВЛЕНЫ РАЗНЫЕ КУЛЬТУРЫ И ГОСУДАРСТВО И ОБЩЕСТВО ДОЛЖНЫ НАУЧИТЬСЯ УПРАВЛЯТЬ ЭТИМ МНОГООБРАЗИЕМ. КОРЕННЫЕ НАРОДЫ ИСПОЛЬЗУЮТ ДЛЯ ЭТОГО НОРМЫ И МЕХАНИЗМЫ СВОЕЙ ТРАДИЦИОННОЙ КУЛЬТУРЫ. СЛОВО И МОЛЧАНИЕ ЯВЛЯЮТСЯ НЕ ЕДИНСТВЕННЫМИ, НО НАИБОЛЕЕ ЯРКИМИ, СУЩНОСТНЫМИ ЗНАКАМИ АБОРИГЕННОЙ КУЛЬТУРЫ, ВАЖНЫМИ ДЛЯ ЕЕ НАУЧНОЙ ИНТЕРПРЕТАЦИИ. КОРЕННЫЕ НАРОДЫ СТРЕМЯТСЯ УСТАНОВИТЬ ПАРИТЕТНЫЕ ОТНОШЕНИЯ И ДОБИТЬСЯ ИХ ПРИНЯТИЯ ГОСУДАРСТВОМ И ПРОМЫШЛЕННЫМИ КОРПОРАЦИЯМИ, ПОКАЗЫВАЯ ПРИ ЭТОМ, ЧТО НА СЕВЕРЕ, НЕСМОТРЯ НА ЕГО ОГРОМНЫЕ ПРОСТРАНСТВА, СУЩЕСТВУЮТ СЛИШКОМ ТЕСНЫЕ СВЯЗИ МЕЖДУ ЛЮДЬМИ, МЕЖДУ ЛЮДЬМИ И ПРИРОДОЙ. МОИ ПОЛЕВЫЕ МАТЕРИАЛЫ ПОКАЗЫВАЮТ, ЧТО АБОРИГЕНЫ ОЧЕНЬ ЦЕНЯТ В ЛЮДЯХ УМЕНИЕ МОЛЧАТЬ, В ДИАЛОГЕ ВЕСТИ СЕБЯ ПРАВИЛЬНО, НЕ ГОВОРЯ ЛИШНЕГО. ТАКОЕ ПОВЕДЕНИЕ ПРЕДПОЛАГАЕТ НЕ ТОЛЬКО ЭМПАТИЮ СОБЕСЕДНИКА, НО И ОТВЕТСТВЕННОСТЬ ЗА СВОИ ДЕЙСТВИЯ И СЛОВА. 


\section{BIBLIOGRAPHIE}

БАЙБУРИН А.К., РИТУАЛ В ТРАДИЦИОННОЙ КУЛЬТУРЕ, СПБ., 1993

БУТОВСКАЯ М.Л., ЯЗЫК ТЕЛА: ПРИРОДА И КУЛЬТУРА, М., 2004

ГИРЦ К., ИНТЕРПРЕТАЦИЯ КУЛЬТУР, М., 2004

ГЛУХИХ, А., «ЛЕНИН ТОЖЕ ОТВЕРНУЛСЯ», НОВОСТИ ЮГРЫ, 11.05.1995

ГОЛОВНЕВ А.В., ГОВОРЯЩИЕ КУЛЬТУРЫ: ТРАДИЦИИ САМОДИЙЦЕВ И УГРОВ, ЕКАТЕРИНБУРГ, 1995.

ГУРЕВИЧ А.Я., «НЕСКОЛЬКО СООБРАЖЕНИЙ НА ПОЛЯХ СТАТЬИ ЭВЫ ЭСТЕРБЕРГ», Arbor Mundi. МИРОВОЕ ДЕРЕВО, 1996, № 4, С. 43-46.

ИВАНОВ С.В., «ДРЕВНИЕ ПРЕДСТАВЛЕНИЯ НЕКОТОРЫХ НАРОДОВ СИБИРИ О СЛОВЕ, МЫСЛИ И ОБРАЗЕ», СТРАНЫ И НАРОДЫ ВОСТОКА, ВЫП. XVII. КН. 3, М., 1975, С. 119-126.

КРЕЙДЛИН Г.Е., МУЖЧИНЫ И ЖЕНЩИНЫ В НЕВЕРБАЛЬНОЙ КОММУНИКАЦИИ, М., 2005

ЛАПИНА М., ЭТИКЕТ ХАНТОВ, ТОМСК, 1998

ЛИч Э., КУЛЬТУРА И КОММУНИКАЦИЯ. ЛОГИКА ВЗАИМОСВЯЗИ СИМВОЛОВ, М., 2001

НОВИКОВА Н.И., ТРАДИЦИОННЫЕ ПРАЗДНИКИ МАНСИ, М., 1995

НОВИКОВА Н.И., «ИНСТИТУТЫ ПРАВОСУДИЯ: ПУТИ ВОССТАНОВЛЕНИЯ СПРАВЕДЛИВОСТИ НА СЕВЕРЕ КАНАДЫ», РАСЫ И НАРОДЫ, ЕЖЕГОДНИК 33, МОСКВА, 2007, С. 111-128

ПЕСИКОВА А.С., «НЕКОТОРЫЕ АСПЕКТЫ ЭТНОПСИХОЛОГИИ ОБСКИХ УГРОВ (НА ПРИМЕРЕ ПИМСКИХ ХАНТЫ)», НАУЧНЫЕ ТРУДЫ СУРГУТСКОГО УНИВЕРСИТЕТА (СУРГУ). СУРГУТ, 1996, ТОМ. 3.

ПЕСИКОВА А.С., «СОВРЕМЕННАЯ СТРУКТУРА МИРОПОНИМАНИЯ ОБСКИХ УГРОВ, ВЕДУЩИХ ТРАДИЦИОННЫЙ ОБРАЗ ЖИЗНИ (НА ПРИМЕРЕ СУРГУТСКИХ ХАНТЫ)», ПАМЯТНИКИ ЮГРЫ, ТОМСК, 2000

ПОПОВ А.А., «ПЕРЕЖИТКИ ДРЕВНИХ ДОРЕЛИГИОЗНЫХ ВОЗЗРЕНИЙ ДОЛГАНОВ НА ПРИРОДУ», СЭ, 1959, №2

ПРАНИС К., СТЮАРТ Б., УЕДЖ М., КРУГИ ПРИМИРЕНИЯ. ОТ ПРЕСТУПЛЕНИЯ К СООБЩЕСТВУ, ПЕР. С АНГЛ., М., 2010

ПУШКАРЕВА Е.Т., «ОБРАЗЫ СЛОВА В ФОЛЬКЛОРЕ НЕНЦЕВ», ЭО, 2002, № 4, С. 28-38

ПУШКАРЕВА Е.Т., КАРТИНА МИРА В ФОЛЬКЛОРЕ НЕНЦЕВ: СИСТЕМНО-ФЕНОМЕНОЛОГИЧЕСКИЙ АНАЛИЗ, ЕКАТЕРИНБУРГ, 2007

ТЭРНЕР В., СИМВОЛ И РИТУАЛ, М., 1983

ХРИСТОФОРОВА О.Б., «ОСОБЕННОСТИ КОММУНИКАТИВНОГО ПОВЕДЕНИЯ НАРОДОВ СИБИРИ И ИХ РОЛЬ В МЕЖКУЛЬТУРНОМ ОБЩЕНИИ», ЯЗЫКОВОЕ СОЗНАНИЕ: ФОРМИРОВАНИЕ И ФУНКЦИОНИРОВАНИЕ, M., 1998, C. 224-231

ХРИСТОФОРОВА О.Б., «ИНТЕРПРЕТАЦИИ МОЛЧАНИЯ: РЕЧЕВОЙ ЭТИКЕТ НАРОДОВ СЕВЕРА В ЗАМЕТКАХ ПУТЕШЕСТВЕННИКОВ И ПО ДАННЫМ ФОЛЬКЛОРА», Arbor mundi. МЕЖДУНАРОДНЫЙ ЖУРНАЛ ПО ТЕОРИИ И ИСТОРИИ МИРОВОЙ КУЛЬТУРЫ, ВЫП. 12. 2006, с. 82-194. 
ЭСТЕРБЕРГ Э., «МОЛЧАНИЕ КАК СТРАТЕГИЯ ПОВЕДЕНИЯ. СОЦИАЛЬНОЕ ОКРУЖЕНИЕ И МЕНТАЛЬНОСТЬ В ИСЛАНДСКИХ САГАХ», Arbor Mundi. МИРОВОЕ ДЕРЕВО, 1996, № 4.

BASSO K., “"To Give up on Words»: Silence in Western Apache Culture”, Southwestern Journal of Anthropology, 1970, № 26. p. 213-230

JENNESS D., The life of The Copper Eskimo, Ottawa, 1923, p. 184

\section{NOTES}

1. ПОЛЕВЫЕ МАТЕРИАЛЫ АВТОРА, ИНТЕРВЬЮ С Г.С. РАЙШЕВЫМ, 2001 Г., Г. ХАНТЫМАНСИЙСК.

2. ГОСУДАРСТВЕННЫЙ АРХИВ ХАНТЫ-МАНСИЙСКОГО АВТОНОМНОГО ОКРУГА, Ф.138, ОП.

1, Д.18, Л. 2-5. 